\title{
Deep observation of the NGC 1275 region with MAGIC: search of diffuse $\gamma$-ray emission from cosmic rays in the Perseus cluster
}

\author{
M. L. Ahnen ${ }^{1}$, S. Ansoldi ${ }^{2}$, L. A. Antonelli ${ }^{3}$, P. Antoranz ${ }^{4}$, A. Babic ${ }^{5}$, B. Banerjee ${ }^{6}$, P. Bangale ${ }^{7}$,
} U. Barres de Almeida ${ }^{7,25}$, J. A. Barrio ${ }^{8}$, J. Becerra González ${ }^{9,26}$, W. Bednarek ${ }^{10}$, E. Bernardini ${ }^{11,27}$, B. Biasuzzi ${ }^{2}$, A. Biland ${ }^{1}$, O. Blanch ${ }^{12}$, S. Bonnefoy ${ }^{8}$, G. Bonnoli ${ }^{3}$, F. Borracci ${ }^{7}$, T. Bretz ${ }^{13,28}$, S. Buson ${ }^{14}$, E. Carmona ${ }^{15}$, A. Carosi ${ }^{3}$,

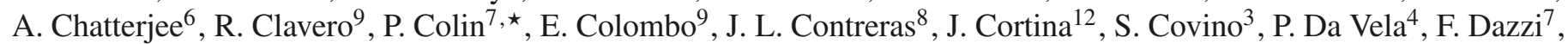
A. De Angelis ${ }^{14}$, B. De Lotto ${ }^{2}$, E. de Oña Wilhelmi ${ }^{16}$, C. Delgado Mendez ${ }^{15}$, F. Di Pierro ${ }^{3}$, A. Domínguez ${ }^{8,29}$,

D. Dominis Prester ${ }^{5}$, D. Dorner ${ }^{13}$, M. Doro ${ }^{14}$, S. Einecke ${ }^{17}$, D. Eisenacher Glawion ${ }^{13}$, D. Elsaesser ${ }^{17}$, A. Fernández-Barral ${ }^{12}$, D. Fidalgo ${ }^{8}$, M. V. Fonseca ${ }^{8}$, L. Font ${ }^{18}$, K. Frantzen ${ }^{17}$, C. Fruck $^{7}$, D. Galindo ${ }^{19}$, R. J. García López ${ }^{9}$, M. Garczarczyk ${ }^{11}$, D. Garrido Terrats ${ }^{18}$, M. Gaug ${ }^{18}$, P. Giammaria ${ }^{3}$, N. Godinović ${ }^{5}$, A. González Muñoz ${ }^{12}$, D. Gora ${ }^{11}$, D. Guberman ${ }^{12}$, D. Hadasch $^{20}$, A. Hahn ${ }^{7}$, Y. Hanabata ${ }^{20}$, M. Hayashida ${ }^{20}$, J. Herrera ${ }^{9}$, J. Hose ${ }^{7}$, D. Hrupec ${ }^{5}$, G. Hughes ${ }^{1}$, W. Idec $^{10}$, K. Kodani ${ }^{20}$, Y. Konno ${ }^{20}$, H. Kubo ${ }^{20}$, J. Kushida ${ }^{20}$, A. La Barbera ${ }^{3}$, D. Lelas ${ }^{5}$, E. Lindfors ${ }^{21}$, S. Lombardi ${ }^{3}$, F. Longo $^{2}$, M. López ${ }^{8}$, R. López-Coto ${ }^{12}$, E. Lorenz ${ }^{7}$, P. Majumdar ${ }^{6}$, M. Makariev ${ }^{22}$, K. Mallot ${ }^{11}$, G. Maneva ${ }^{22}$, M. Manganaro ${ }^{9}$, K. Mannheim ${ }^{13}$, L. Maraschi ${ }^{3}$, B. Marcote ${ }^{19}$, M. Mariotti ${ }^{14}$, M. Martínez ${ }^{12}$, D. Mazin ${ }^{7,30}$, U. Menzel ${ }^{7}$, J. M. Miranda ${ }^{4}$, R. Mirzoyan ${ }^{7}$, A. Moralejo ${ }^{12}$, E. Moretti ${ }^{7}$, D. Nakajima ${ }^{20}$, V. Neustroev ${ }^{21}$, A. Niedzwiecki ${ }^{10}$, M. Nievas Rosillo ${ }^{8}$, K. Nilsson ${ }^{21,31}$, K. Nishijima ${ }^{20}$, K. Noda ${ }^{7}$, R. Orito ${ }^{20}$, A. Overkemping ${ }^{17}$, S. Paiano ${ }^{14}$, J. Palacio ${ }^{12}$, M. Palatiello ${ }^{2}$, D. Paneque ${ }^{7}$, R. Paoletti ${ }^{4}$, J. M. Paredes ${ }^{19}$, X. Paredes-Fortuny ${ }^{19}$, G. Pedaletti ${ }^{11}$, M. Persic ${ }^{2,32}$, J. Poutanen ${ }^{21}$, P. G. Prada Moroni ${ }^{23}$, E. Prandini ${ }^{1,33}$, I. Puljak ${ }^{5}$, W. Rhode ${ }^{17}$, M. Ribó ${ }^{19}$, J. Rico ${ }^{12}$, J. Rodriguez Garcia ${ }^{7}$, T. Saito ${ }^{20}$, K. Satalecka ${ }^{8}$, C. Schultz ${ }^{14}$, T. Schweizer ${ }^{7}$, A. Sillanpää2 ${ }^{21}$, J. Sitarek ${ }^{10}$, I. Snidaric ${ }^{5}$, D. Sobczynska ${ }^{10}$, A. Stamerra ${ }^{3}$, T. Steinbring ${ }^{13}$,

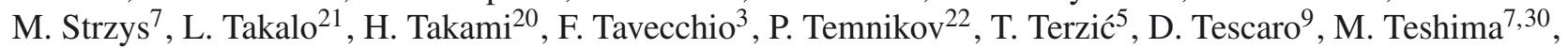
J. Thaele ${ }^{17}$, D. F. Torres ${ }^{24}$, T. Toyama ${ }^{7}$, A. Treves ${ }^{2}$, M. Vazquez Acosta ${ }^{9}$, V. Verguilov ${ }^{22}$, I. Vovk ${ }^{7}$, J. E. Ward ${ }^{12}$, M. Will ${ }^{9}$, M. H. Wu ${ }^{16}$, R. Zanin ${ }^{19}$ (The MAGIC Collaboration) and C. Pfrommer ${ }^{34}$, A. Pinzke ${ }^{35}$, and F. Zandanel ${ }^{36, \star}$

(Affiliations can be found after the references)

Received 25 November 2015 / Accepted 4 February 2016

\section{ABSTRACT}

Clusters of galaxies are expected to be reservoirs of cosmic rays (CRs) that should produce diffuse $\gamma$-ray emission due to their hadronic interactions with the intra-cluster medium. The nearby Perseus cool-core cluster, identified as the most promising target to search for such an emission, has been observed with the MAGIC telescopes at very-high energies (VHE, $E \gtrsim 100 \mathrm{GeV}$ ) for a total of $253 \mathrm{~h}$ from 2009 to 2014 . The active nuclei of NGC 1275, the central dominant galaxy of the cluster, and IC 310, lying at about $0.6^{\circ}$ from the centre, have been detected as point-like VHE $\gamma$-ray emitters during the first phase of this campaign. We report an updated measurement of the NGC 1275 spectrum, which is described well by a power law with a photon index $\Gamma=3.6 \pm 0.2_{\text {stat }} \pm 0.2_{\text {syst }}$ between $90 \mathrm{GeV}$ and $1200 \mathrm{GeV}$. We do not detect any diffuse $\gamma$-ray emission from the cluster and so set stringent constraints on its CR population. To bracket the uncertainties over the CR spatial and spectral distributions, we adopt different spatial templates and power-law spectral indexes $\alpha$. For $\alpha=2.2$, the CR-to-thermal pressure within the cluster virial radius is constrained to be $\$ 1-2 \%$, except if CRs can propagate out of the cluster core, generating a flatter radial distribution and releasing the CR-to-thermal pressure constraint to $\lesssim 20 \%$. Assuming that the observed radio mini-halo of Perseus is generated by secondary electrons from CR hadronic interactions, we can derive lower limits on the central magnetic field, $B_{0}$, that depend on the CR distribution. For $\alpha=2.2, B_{0} \gtrsim 5-8 \mu \mathrm{G}$, which is below the $\sim 25 \mu \mathrm{G}$ inferred from Faraday rotation measurements, whereas for $\alpha \lesssim 2.1$, the hadronic interpretation of the diffuse radio emission contrasts with our $\gamma$-ray flux upper limits independently of the magnetic field strength.

Key words. gamma rays: galaxies: clusters - acceleration of particles - galaxies: clusters: individual: Perseus - galaxies: individual: NGC 1275 galaxies: individual: NGC 1265

\section{Introduction}

Clusters of galaxies represent the latest stage of structure formation, and are presently assembling through mergers of

^ Corresponding author: P. Colin, e-mail: colin@mppmu.mpg.de and F. Zandanel, e-mail: f.zandanel@uva.nl smaller groups of galaxies and gas accretion. They are powerful cosmological tools for testing the evolution of the Universe 
(see Voit 2005, for a review). Cosmic-ray (CR) protons can accumulate in clusters of galaxies for cosmological times, accelerated by structure formation shocks, and outflows from active galactic nuclei (AGNs) and galaxies (see, e.g., Völk et al. 1996; Berezinsky et al. 1997; see Brunetti \& Jones 2014, for a review). These CR protons can interact hadronically with the protons of the intra-cluster medium (ICM), a hot thermal plasma $\left(k_{\mathrm{B}} T \simeq 1-10 \mathrm{keV}\right)$ filling the cluster volume, and can generate pions. While the charged pions decay to secondary electrons and neutrinos, the neutral pions decay directly to high-energy $\gamma$ rays. Despite many observational efforts in the past decade, $\gamma$-ray emission from clusters of galaxies remains elusive ${ }^{1}$.

Non-thermal emission is observed at radio frequencies in many clusters of galaxies in the form of diffuse synchrotron radiation (see Feretti et al. 2012, for a review). This probes for the presence of relativistic CR electrons and magnetic fields in the cluster environment. However, a conclusive proof of CR-proton acceleration has yet to be found. The observed CR electrons can also produce hard X-rays by inverse-Compton (IC) scattering of cosmic microwave background (CMB) photons. Several claims of IC detection have been made in the past (see Rephaeli et al. 2008 for a review), but more recent observations do not confirm them (Ajello et al. 2009, 2010; Wik et al. 2011, 2012, 2014; Gastaldello et al. 2015), and the possible diffuse IC emission from clusters remains elusive, too.

The observed diffuse radio emission in clusters can be divided in two main categories: peripheral radio relics and central radio halos (e.g., Feretti et al. 2012; Brunetti \& Jones 2014). The latter are usually divided in two other categories: gianthalos hosted in merging non-cool-core clusters (e.g., the Coma cluster; Deiss et al. 1997; Brown \& Rudnick 2011), and minihalos hosted in relaxed cool-core clusters (e.g., the Perseus cluster; Pedlar et al. 1990; Sijbring 1993; Gitti et al. 2002). While radio relics can be roughly related to merger shocks due to their spatial coincidence and morphology, the explanation for the origin of radio halos is more challenging. The generation mechanism of radio halos has been historically debated between re-acceleration ${ }^{2}$ and hadronic models ${ }^{3}$. In the re-acceleration model, a seed population of CR electrons can

\footnotetext{
For space-based cluster observations in the GeV-band, see Reimer et al. (2003), Fermi-LAT Collaboration (2010a,b), Jeltema \& Profumo (2011), Han et al. (2012), Ando \& Nagai (2012), Huber et al. (2013), Zandanel \& Ando (2014), Fermi-LAT Collaboration (2014), Prokhorov \& Churazov (2014), Vazza \& Brüggen (2014), Griffin et al. (2014), Selig et al. (2015), Vazza et al. (2015), Fermi-LAT Collaboration (2016, 2015). For ground-based observations in the energy band above $100 \mathrm{GeV}$, see Perkins et al. (2006), Perkins (2008), HESS Collaboration (2009a,b), Domainko et al. (2009), Galante et al. (2009), Kiuchi et al. (2009), VERITAS Collaboration (2009), MAGIC Collaboration (2010b, 2012a), VERITAS Collaboration (2012), HESS Collaboration (2012).

2 See, e.g., Schlickeiser et al. (1987), Brunetti et al. (2001), Petrosian (2001), Gitti et al. (2002), Ohno et al. (2002), Fujita et al. (2003), Brunetti et al. (2004), Brunetti \& Blasi (2005), Cassano \& Brunetti (2005), Brunetti \& Lazarian (2007, 2011), Brunetti et al. (2012), Donnert et al. (2013), ZuHone et al. (2013), Pinzke et al. (2015), Miniati (2015), Bravi et al. (2016).

3 See, e.g., Dennison (1980), Vestrand (1982), Enßlin et al. (1997), Blasi \& Colafrancesco (1999), Dolag \& Enßlin (2000), Miniati et al. (2001), Miniati (2003), Pfrommer \& Enßlin (2003), Gabici \& Blasi (2003), Pfrommer \& Enßlin (2004a,b), Blasi et al. (2007), Pfrommer et al. (2008), Pfrommer (2008), Kushnir et al. (2009), Donnert et al. (2010a,b), Keshet \& Loeb (2010), Pinzke \& Pfrommer (2010), Pinzke et al. (2011), Enßlin et al. (2011), Fujita \& Ohira (2012), Zandanel et al. (2014), ZuHone et al. (2015).
}

be re-accelerated by interacting turbulent waves, while in the hadronic scenario, the radio-emitting electrons are secondaries produced by CR protons interacting with the protons of the ICM. Currently, the re-acceleration scenario is favoured as the generation mechanism for giant radio halos, while for mini-halos, both the re-acceleration and hadronic models can explain the observed emission (see, e.g., Enßlin et al. 2011; and Brunetti $\&$ Jones 2014, for extensive discussions).

The key questions are the following. What is the origin of the radio-emitting electrons? What is the role of CR protons and how do they affect the cluster environment? Upcoming X-ray observations have the potential to detect IC emission in clusters and, hopefully, to break the degeneracy between the electron and the magnetic-field distributions (Kitayama et al. 2014; Bartels et al. 2015), providing an alternative estimate for the magnetic field in clusters with respect to Faraday rotation (FR) measurements (Kim et al. 1991; Clarke et al. 2001; Carilli \& Taylor 2002; Vogt \& Enßlin 2005; Bonafede et al. 2010, 2013; Kuchar \& Enßlin 2011). However, the presence and role of CR protons in clusters can only be probed directly through the $\gamma$ rays and neutrinos induced by hadronic interactions. The high-energy astronomy window is then crucial for understanding non-thermal phenomena in clusters of galaxies. (This is also true, albeit even more challenging, for neutrinos; see, e.g., Murase et al. 2008; Zandanel et al. 2015.)

The Perseus cluster of galaxies (a.k.a. Abell 426) is a relaxed cool-core cluster located at a distance of about $D_{\text {lum }}=78 \mathrm{Mpc}$ (redshift $z=0.018$ ). It hosts the brightest thermal ICM in X-rays (Reiprich \& Böhringer 2002) and a very luminous radio mini-halo (Pedlar et al. 1990; Sijbring 1993; Gitti et al. 2002). The high ICM density at the centre of the cluster (Churazov et al. 2003) implies a high density of target protons for hadronic interactions with CR protons. Therefore, Perseus is the best cluster for searching for CR-induced $\gamma$-ray emission (we refer the reader to Pinzke \& Pfrommer 2010; MAGIC Collaboration 2010b, 2012a; Pinzke et al. 2011, for a detailed argumentation). The Perseus cluster also hosts three bright radio galaxies (Ryle \& Windram 1968): NGC 1275, the central dominant galaxy of the cluster, NGC 1265, archetype of a head-tail radio galaxy, in which the jets are bent by their interaction with the ICM, and IC 310, a peculiar object that shows properties of different classifications and which could be an intermediate state between a BL Lac and a radio galaxy. The AGNs of both NGC 1275 and IC 310 show a bright and variable $\gamma$-ray emission in the energy ranges of both the Fermi Large Area Telescope (LAT; Neronov et al. 2010; Fermi-LAT Collaboration 2009) and the atmospheric-Cherenkov telescopes (MAGIC Collaboration 2010a, 2012b, 2014a,b). The NGC 1275 AGN emission obscures the expected diffuse cluster emission over most of the $\gamma$-ray band, in particular in the GeV region, where the spectral energy distribution is peaking (MAGIC Collaboration 2014a).

Since 2008, the Perseus cluster has been intensively observed by the Major Atmospheric Gamma Imaging Cherenkov (MAGIC) telescopes. In this paper we present the results obtained with $\sim 250 \mathrm{~h}$ of effective observation time taken in stereoscopic mode from 2009 to 2014 and derive tight constraints on the CR population in the cluster. We discuss neither the AGN physics nor the possible indirect detection of dark matter from clusters (Palacio et al. 2015). After describing the observation and data analysis in Sect. 2, we report our observational results and searches for a CR-induced signal in Sects. 3 and 4, respectively. In Sect. 5, we discuss the interpretation of our constraints on the CR physics in galaxy clusters, and, finally, in Sect. 6 
we present our conclusions. Throughout the paper, we assume a standard $\Lambda$ CDM cosmology with $H=70 \mathrm{~km} \mathrm{~s}^{-1} \mathrm{Mpc}^{-1}$.

\section{MAGIC observations and data analysis}

MAGIC is a system of two $17 \mathrm{~m}$ diameter imaging atmospheric Cherenkov telescopes located on the Canary island of La Palma, which observes the $\gamma$-ray sky from $\sim 50 \mathrm{GeV}$ to more than $50 \mathrm{TeV}$. The observation of the Perseus cluster started in 2008 with $\sim 24 \mathrm{~h}$ of observation with a single telescope, which did not allow any detection (MAGIC Collaboration 2010b). Since 2009, MAGIC operates in stereoscopic mode, which provides much better sensitivity (about a factor 2 above $300 \mathrm{GeV}$ ). A major upgrade of the telescopes then occurred during the northernhemisphere summers of 2011 and 2012 (MAGIC Collaboration 2016a). The improvement in the MAGIC performance after this upgrade is reported by the MAGIC Collaboration (2016b). Here, we combine all the stereoscopic data taken from October 2009 to November 2014. The observations carried out before the upgrade, from October 2009 to February 2011, led to the detection of IC 310 (MAGIC Collaboration 2010a) and NGC 1275 (MAGIC Collaboration 2012b). These data were taken solely during dark time at a low zenith angle (from $12^{\circ}$ to $36^{\circ}$ ), ensuring the lowest possible energy threshold $(\sim 100 \mathrm{GeV}$ at the analysis level). After data-quality selection, this first sample consists of $85 \mathrm{~h}$ of effective time. The observations carried out after the upgrade from November 2012 to November 2014 were performed under heterogeneous conditions, including moonlight and large-zenith angles (from $12^{\circ}$ to $60^{\circ}$ ), in order to accumulate the largest amount of data possible and improve the performance at $\mathrm{TeV}$ energies. After data-quality selection, the second sample consists of $168 \mathrm{~h}$ of effective time.

All the observations were taken in so-called "wobble" mode, pointing to alternative sky positions lying $0.4^{\circ}$ from NGC 1275 , which is at the centre of the cluster. Most of the pointings were also separated by $0.4^{\circ}$ from IC 310 to allow the survey of both AGNs simultaneously. The standard MAGIC Analysis and Reconstruction Software (MARS, Zanin et al. 2013) was used to analyse the data. The results of the 2009-2011 campaign were already reported by the MAGIC Collaboration (2012a). Here, we re-analyse these data with same calibration, image cleaning, and gamma-hadron separation as previously and combine them with the more recent data only at the last stage of the analysis. For the 2012-2014 data sample, we use the so-called "sum image cleaning" with cleaning thresholds that are $33 \%$ higher than the standard cleaning (reported in MAGIC Collaboration 2016 b) to properly handle the data taken under moonlight conditions. This increases the energy threshold from $100 \mathrm{GeV}$ to about $150 \mathrm{GeV}$ but does not affect the performance at $\mathrm{TeV}$ energies. Additionally, only events with more than 150 photo electrons in the camera image of both telescopes are kept. Below this cut our simulation of the instrument response with fixed pixel discriminator thresholds does not correctly describe the actual MAGIC trigger, which uses adaptive thresholds, during Moon observation.

The gamma-hadron separation is performed by the standard MAGIC method using the Random Forest algorithm (MAGIC Collaboration 2008). The remaining background from the CR-induced air showers is estimated from background control regions (OFF regions) lying at $0.4^{\circ}$ from the camera centre. To prevent contamination by the strong IC 310 signal, the OFF regions closer than $0.4^{\circ}$ to IC 310 have been excluded. Our analysis assumes different source extensions that only differ on the signal-region radius, $\theta_{\text {cut }}$, and the number of OFF regions.
For the point-like source analysis, an average of 5 OFF regions in the field of view are used. In the case of extended source analysis, only the most distant $\mathrm{OFF}$ region, lying $0.8^{\circ}$ from NGC 1275, is used.

\section{MAGIC results}

The $253 \mathrm{~h}$ of stereo observation from different periods were combined to provide the deepest view of the Perseus cluster at very high energy. The upper left- and right-hand panels of Fig. 1 show the relative-flux (i.e., signal-to-background ratio) sky maps for an energy threshold of $250 \mathrm{GeV}$ and $1 \mathrm{TeV}$, respectively. A clear signal is detected from the two previously discovered AGNs. The bright and hard source IC 310 is visible in both maps with a high significance, and it could mask smaller signals. To search for weak emissions, we modelled the point-like emission from IC 310 as an additional background component. The lower panels of Fig. 1 show the resulting significance sky maps. Above $250 \mathrm{GeV}$, the emission from NGC 1275 is detected with a significance of $8.5 \sigma$ and a signal-to-background ratio greater than $20 \%$. Above $1 \mathrm{TeV}$, however, no source other than IC 310 is detected. Figure 2 compares the excess ( $\gamma$-ray) event distribution above $250 \mathrm{GeV}$ as a function of the squared distance from NGC $1275\left(\theta^{2}\right)$ with the MAGIC point spread function (PSF) obtained from contemporaneous Crab nebula data at similar zenith angles. In this $\theta^{2}$ plot, the PSF is described by a double exponential (corresponding to a double Gaussian in a 2D map), which matches the excess shape of the Crab nebula ${ }^{4}$ well (MAGIC Collaboration 2016b). The shape of the detected signal is in perfect agreement with a point-like object such as the one expected for an AGN. At both energies, there is no sign of diffuse $\gamma$-ray structures inside the cluster.

The average energy spectrum of NGC 1275 obtained with the full data set (August 2009-November 2014) is shown in Fig. 3, together with the previous results from the first two years of observation (MAGIC Collaboration 2014a). The new spectrum starts at higher energy because the data include moonlight and large-zenith-angle observations. With increased photon statistics, we get better precision and reach higher energies up to $880 \mathrm{GeV}$. This last data point is only marginally significant $(\sim 2 \sigma)$ and in agreement with the upper limits discussed later. The spectrum between $90 \mathrm{GeV}$ and $1200 \mathrm{GeV}$ can be described well by a simple power law ${ }^{5}$

$$
\frac{\mathrm{d} F}{\mathrm{~d} E}=f_{0}\left(\frac{E}{200 \mathrm{GeV}}\right)^{-\Gamma},
$$

with a photon index $\Gamma=3.6 \pm 0.2_{\text {stat }} \pm 0.2_{\text {syst }}$ and a normalisation constant at $200 \mathrm{GeV}$ of $f_{0}=\left(2.1 \pm 0.2_{\text {stat }} \pm 0.3_{\text {syst }}\right) \times$ $10^{-11} \mathrm{~cm}^{-2} \mathrm{~s}^{-1} \mathrm{TeV}^{-1}$. The systematic errors on the flux normalisation and spectral slope take the signal-to-noise ratio into account as explained in MAGIC Collaboration (2016b). Additionally, the uncertainty on the energy scale is $15 \%$.

The average NGC 1275 spectrum from August 2009 to November 2014 is in good agreement with previous measurements (MAGIC Collaboration 2014a). The transition between a flat spectrum, $\Gamma \simeq 2$, measured by Fermi-LAT in $0.1-10 \mathrm{GeV}$

\footnotetext{
4 The Crab nebula has a much harder spectrum than NGC 1275, so the PSF for NGC 1275 could be slightly larger. The PSF shown in Fig. 2 has been normalised to fit the NGC 1275 signal. It is just illustrative and no quantitative result on the intrinsic extension can be derived.

5 Power-law fit obtained with the forward-unfolding method over 7 reconstructed-energy bins $\left(\chi^{2} / n_{\text {d.o.f. }}=2.4 / 5\right)$.
} 

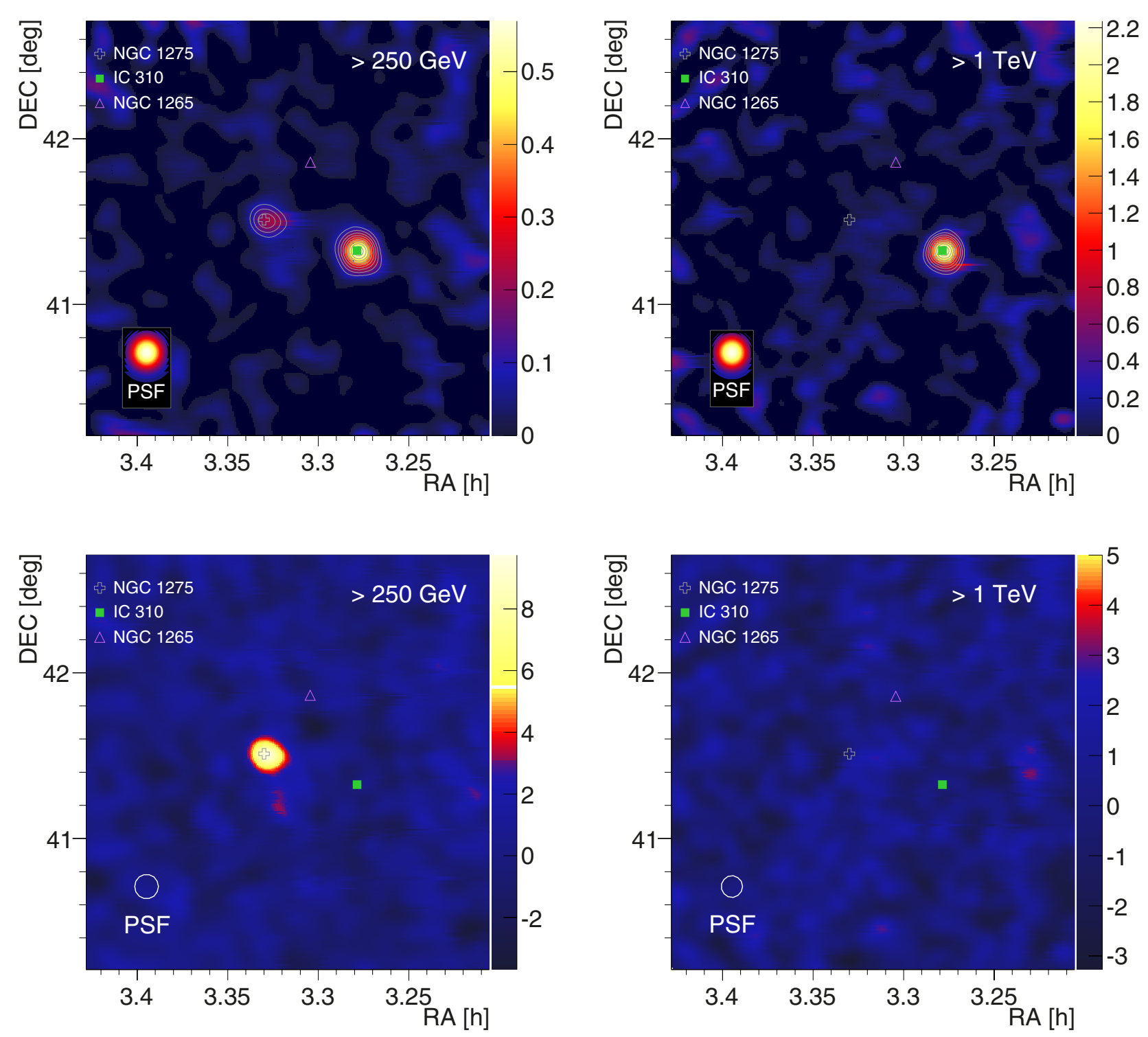

Fig. 1. Perseus cluster sky maps for an energy threshold of $250 \mathrm{GeV}$ (left-hand panels) and $1 \mathrm{TeV}$ (right-hand panels) obtained from $253 \mathrm{~h}$ of MAGIC observation. Upper panels show the relative flux (colour code, expressed in signal-to-background ratio) and the excess significance (contour lines starting from $4 \sigma$ with steps of $2 \sigma$ ). Lower panels show the significance maps where the signal from IC 310 is subtracted (see text). Symbols indicate the positions of the three brightest radio galaxies of the cluster.

range, and the soft spectrum observed above $100 \mathrm{GeV}$ is confirmed to be smooth, and better fitted by a log parabola or a broken power law compared to a cut-off hypothesis (see discussion in MAGIC Collaboration 2014a). The AGN-physics interpretation is not substantially modified with respect to our previous results.

Figure 3 also reports the differential flux upper limits of four energy bins above $1 \mathrm{TeV}$ for a point-like source with a soft power-law spectrum $(\Gamma=3.5)$ as measured in the $0.1-1 \mathrm{TeV}$ range, and for a hard spectral index $(\Gamma=2.3)$, as approximately expected from the CR-induced signal (see next section). Upper limits are calculated using the method of Rolke et al. (2005) for a confidence level (c.1.) of $95 \%$ and with a total systematic uncertainty of $30 \%$. These results assume a point-like emission, while the CR-induced signal should be spatially extended. Upper limits on such diffuse emission depend on the assumption of the surface brightness shape. In the next section, we discuss several CR-induced emission models and report the associated flux upper limits.
The non-detection of NGC 1265 in Fig. 1 allows us to derive flux upper limits for this radio galaxy. Also, $\gamma$ rays could be expected from the central AGN or from the bowshock (Sijbring \& de Bruyn 1998) as speculated earlier for IC 310 before the flux variability was established, confirming the AGN nature of the emission (Neronov et al. 2010). The NGC 1265 position is slightly off-centred, and the exposure at this position is about $20 \%$ lower than for NGC 1275. Assuming a point-like source with the same spectral shape as NGC 1275 (a power law with photo index $\Gamma=3.6$ ), the upper limit of the integral flux above $250 \mathrm{GeV}$ is estimated to be $5.6 \times 10^{-13} \mathrm{~cm}^{-2} \mathrm{~s}^{-1}$.

\section{Search for cosmic-ray induced emission}

As discussed in Sect. 3, the $\gamma$-ray emission from the central galaxy NGC 1275 is consistent with a point-like source, and no diffuse component seems to be present. The measured flux around $100 \mathrm{GeV}$ is much larger than what is expected from the CR-induced emission, and it could be outshining the latter. 
M. L. Ahnen et al.: Search of diffuse $\gamma$-ray emissions from cosmic rays in the Perseus cluster

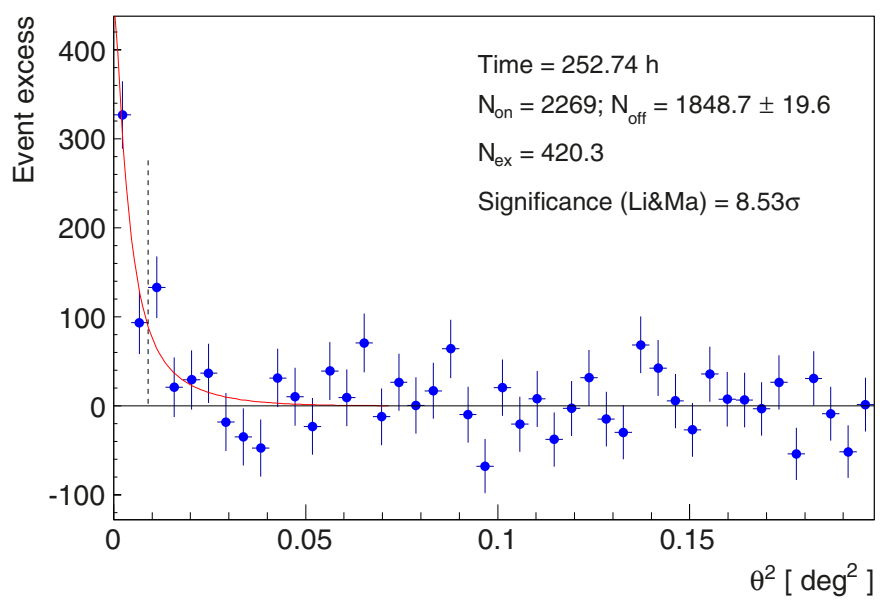

Fig. 2. Excess signal above $250 \mathrm{GeV}$ as a function of the squared distance to NGC $1275\left(\theta^{2}\right)$. The solid red line is the MAGIC PSF fit, and the vertical dashed line shows the optimal $\theta^{2}$ cut used for a point-like source detection.

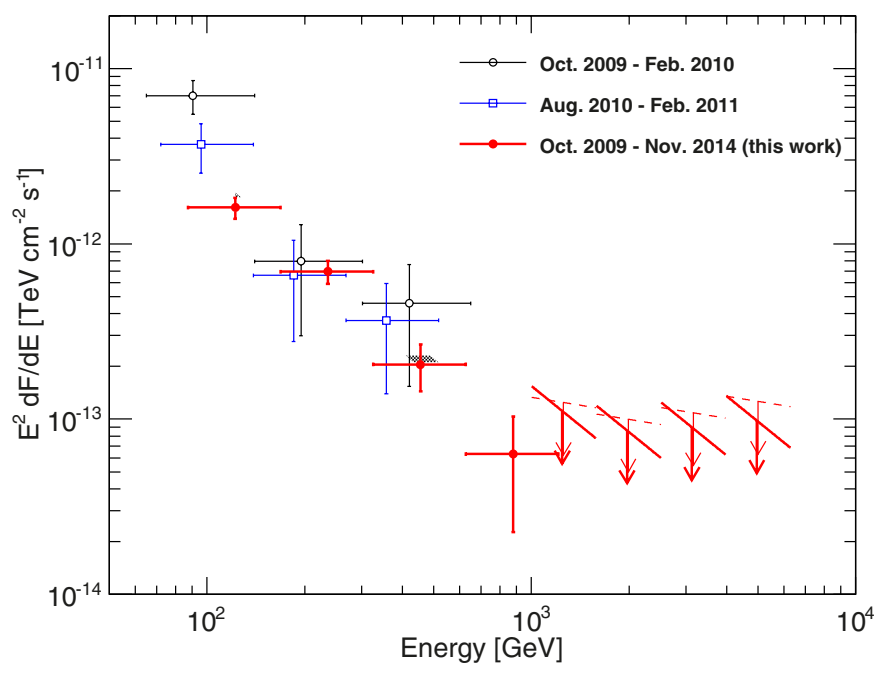

Fig. 3. Spectral energy distribution of NGC 1275 averaged over different periods with the newest results in red $(\sim 250 \mathrm{~h})$. Arrows indicate the 95\%-c.l. differential flux upper limits (5 bins/decade) for a power-law spectrum with a photon index of $\Gamma=3.5$ (thick solid lines) and $\Gamma=2.3$ (thin and dashed lines), respectively.

However, the NGC 1275 AGN spectrum is very steep, and no signal is detected above about $1 \mathrm{TeV}$. The CR-induced emission should be harder with no intrinsic cut-off in the MAGIC energy range ${ }^{6}$. Therefore, we use the energies above $1 \mathrm{TeV}$ to search for the possible diffuse CR-induced component.

In this section, we discuss the expected energy spectrum and spatial distribution of the CR-induced $\gamma$-ray signal as it should be observed by MAGIC, when taking the absorption during the travel to Earth and the instrument response into account. Then, we present the optimisation of our analysis to detect such emissions and the derived flux upper limits.

\footnotetext{
6 A cut-off is expected at PeV energies owing to the escape of highenergy CR protons that are no longer confined in the cluster volume (see, e.g., Völk et al. 1996; Berezinsky et al. 1997; Pinzke \& Pfrommer 2010).
}

\subsection{Spectrum expected on Earth}

The hydrodynamical simulations of Pinzke \& Pfrommer (2010) suggest a universal CR-proton momentum spectrum $p^{-\alpha}$ with $\alpha \approx 2.2$ at the energies of interest here. The very high-energy $\gamma$-ray spectrum induced by pion decays from CR-ICM interactions should have approximately the same spectral index as the CR spectrum. However, these $\gamma$ rays can interact by pair production with the extragalactic background light (EBL) during their travel to Earth, reducing the observed flux. To convert the intrinsic spectrum from the Perseus cluster at $z=0.018$ into the spectrum observed on Earth, we use the EBL model of Domínguez et al. (2011). Below $300 \mathrm{GeV}$, the EBL absorption is negligible $(<4 \%)$. Between $300 \mathrm{GeV}$ and $10 \mathrm{TeV}$, the effect of the absorption can be approximated by an increase (softening) in the power-law index of about 0.13 and a reduction of the differentialflux normalisation at $1 \mathrm{TeV}$ of $17 \%$. Above $10 \mathrm{TeV}$, the absorption increases dramatically. A source with an intrinsic power-law spectrum index of $\alpha=2.2$ would appear above $300 \mathrm{GeV}$ as a power law with an index $\Gamma=2.33$ and a cut-off above $10 \mathrm{TeV}$. About $20 \%$ of the flux above $1 \mathrm{TeV}$ and $60 \%$ above $10 \mathrm{TeV}$ is absorbed during the travel to Earth.

There are three different scenarios for the fate of the ultra-relativistic electron-positron pairs that are produced by $\gamma$-ray-EBL interactions. Each possibility predicts a different signal for the energy range of interest here.

1. The pairs can interact with $\mathrm{CMB}$ photons that are Compton up-scattered in energy. As a result, an initial $\mathrm{TeV} \gamma$-ray is reprocessed to $\mathrm{GeV}$ energies. If the initial $\gamma$ ray has a much higher energy, this process can be repeated to produce the so-called inverse Compton cascade (ICC) effect (see, e.g., Neronov \& Vovk 2010). To first approximation, the ICC emission into the energy range of interest here, $\sim 1-10 \mathrm{TeV}$, is induced by primary $\gamma$ rays from the energy range $\sim 30-100 \mathrm{TeV}$. Assuming the most favourable case, when all the absorbed energy is re-emitted, the ICC emission from a power-law spectrum with $\alpha=2.2$ would represent about $30 \%$ of the intrinsic emission of the $\sim 1-10 \mathrm{TeV}$ range.

2. The compact ICC signal could be diluted by the presence of extragalactic magnetic field (EGMF), which deflects the electron and positron tracks and, consequently, induces angular dispersion, as well as a time delay of the signal (see, e.g., Neronov \& Vovk 2010; Tavecchio et al. 2010, 2011; Dermer et al. 2011; Taylor et al. 2011; Dolag et al. 2011; Takahashi et al. 2012). Being constant, the CR-induced emission is not affected by the time delay. According to the approximation of Neronov \& Vovk (2010), the ICC emission induced by $50 \mathrm{TeV} \gamma$ rays from $78 \mathrm{Mpc}$ should have a spatial extension of the order of $0.1^{\circ}$ for an EGMF $\approx 10^{-14} \mathrm{G}$. For a weaker EGMF, the spatial distribution of the ICC would be almost the same as the intrinsic CR-induced emission, and one could expect up to $30 \%$ higher signal in the $\sim 1-10 \mathrm{TeV}$ range (assuming the intrinsic power-law spectrum extends to $100 \mathrm{TeV})$. For an EGMF $\gg 10^{-14} \mathrm{G}$, however, the ICC would be diluted in the diffuse extragalactic $\gamma$-ray background. Thus, depending on the EGMF level, the $\gamma$-ray loss due to the EBL absorption in the $\sim 1-10 \mathrm{TeV}$ range could be compensated for by the ICC emission, up to a full compensation for a very low EGMF.

3. A competing mechanism exists that could modify the evolution of the pairs on a faster time scale than the ICC, namely powerful plasma instabilities driven by the anisotropy of 
the ultra-relativistic pair beams (Broderick et al. 2012; Schlickeiser et al. 2012a,b, 2013; Chang et al. 2012; Pfrommer et al. 2012; Miniati \& Elyiv 2013; Sironi \& Giannios 2014; Supsar \& Schlickeiser 2014; Chang et al. 2014). This picture is interesting because it can match the observed extragalactic $\gamma$-ray background spectrum above $3 \mathrm{GeV}$ and flux distributions of TeV blazars simultaneously, using a unified model of AGN evolution (Broderick et al. 2014a,b). In contrast to the set-up studied by Broderick et al. (2012), here, we have to compare the oblique instability growth rate, $\Gamma_{\mathrm{M}, \mathrm{k}} \propto n_{\text {beam }}$ (where $n_{\text {beam }}$ is density of beam pairs), to the IC cooling rate, $\Gamma_{\text {IC }}$, at a fixed distance to the source. The mean-free-path to pair production, $d_{\mathrm{pp}}$, of primary $\gamma$ rays with energy $>15 \mathrm{TeV}$ is smaller than $D_{\text {lum }}=78 \mathrm{Mpc}$, the distance of the Perseus cluster. Hence, the density of beam pairs is lower at $D_{\text {lum }}$ in comparison to $d_{\mathrm{pp}}$. As the expected cluster luminosity is smaller than the minimum luminosity of $E L_{E, \min } \approx 10^{42} \mathrm{erg} \mathrm{s}^{-1}$ (at $z \approx 0$ and $d_{\mathrm{pp}}$ from the source) needed for the oblique instability to grow faster than IC cooling rate, $\Gamma_{\mathrm{M}, \mathrm{k}}<\Gamma_{\mathrm{IC}}$ at $d_{\mathrm{pp}}$ (a fortiori at $\left.D_{\text {lum }}\right)$, the absorbed $30-100 \mathrm{TeV} \gamma$-ray flux is very likely reprocessed via ICCs to our energy range of interest.

The EBL effect was neglected in all previous papers on this topic, for which, therefore, the constraints derived from $\gamma$-ray flux upper limits above $\sim 1 \mathrm{TeV}$ can be considered as optimistic. They correspond roughly to the case of a strong ICC emission $\left(\right.$ EGMF $<10^{-14} \mathrm{G}$ ), which compensates for the EBL absorption. Here, in contrast, we include EBL absorption in our reference case and assume the most conservative case without ICC emission. Also, CR propagation effects, such as streaming and diffusion, could cause a softening of the CR spectrum. In the following we do not consider this possibility because of the rather uncertain modelling at this stage (e.g., Wiener et al. 2013).

\subsection{Flux upper limits for different spatial distributions}

To account for our limited knowledge of the spatial shape of the CR-induced emission, we adopted three different models that describe the CR density as a function of the distance from the cluster centre: i) the isobaric model assuming a constant CR-tothermal pressure $X_{\mathrm{CR}}=P_{\mathrm{CR}} / P_{\text {th }}$ (Pfrommer \& Enßlin 2004a); ii) the semi-analytical model of Pinzke \& Pfrommer (2010) derived from hydrodynamical simulations of clusters; and iii) the extended hadronic model of Zandanel et al. (2014), where the possibility of CR propagation out of the cluster core is considered, resulting in a significantly flatter CR profile ${ }^{7}$. In Fig. 4, we show the $\gamma$-ray surface brightness above $100 \mathrm{GeV}$ for the three models. In all cases, fundamental input parameters are the Perseus ICM density and temperature as measured in X-rays (Churazov et al. 2003). The fraction of signal expected within a circular region of a given radius $\theta$ from the cluster centre is shown in Fig. 5, together with the MAGIC PSF above $630 \mathrm{GeV}$.

Since the predicted CR-induced signal extension is significantly larger than the PSF, the optimal $\theta_{\text {cut }}$ used to detect the emission is different than for a point-like source. Comparing the predicted signal in the ON region $\left(\theta<\theta_{\text {cut }}\right)$ with the corresponding background level estimated from our data, and taking the expected signal leakage inside the OFF region at $0.8^{\circ}$ from the cluster centre into account, we optimised the $\theta_{\text {cut }}$ for each model.

\footnotetext{
7 The extended model adopted in this work corresponds to the model with $\gamma_{\mathrm{tu}}=3$ in Zandanel et al. (2014), where $\gamma_{\mathrm{tu}}$ is a parameter that indicates the dominant CR transport mechanism.
}

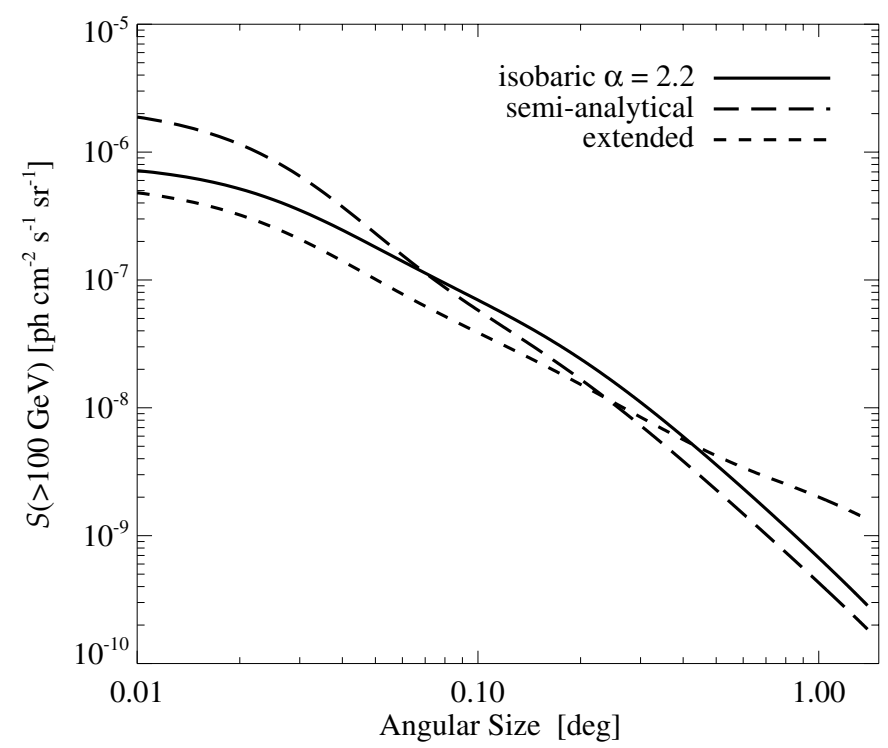

Fig. 4. Surface brightness profiles, above $100 \mathrm{GeV}$, of the three tested spatial templates for the CR-induced emission in Perseus: isobaric with $\alpha=2.2$, semi-analytical, and extended. The normalisation of the isobaric model is set to respect our previous upper limits (MAGIC Collaboration 2012a), while the normalisations of the semi-analytical and extended models are as from Pinzke \& Pfrommer (2010) and Zandanel et al. (2014), respectively. See main text for details.

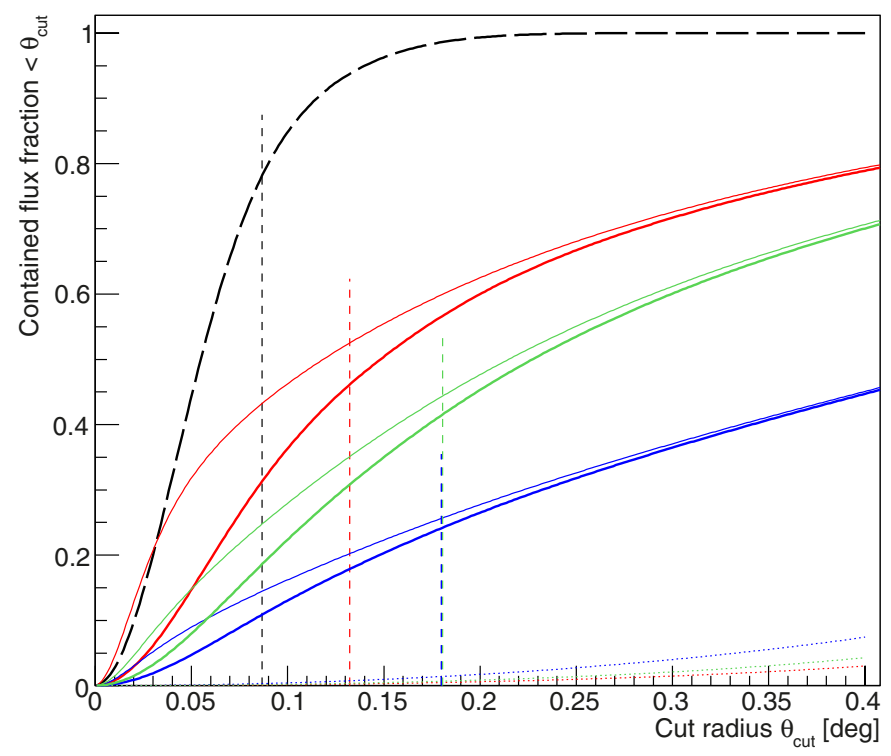

Fig. 5. Cumulative fraction of signal within a given radius for different models: point-like in black (long-dashed line), isobaric in green, semianalytical in red, and extended in blue. The distribution of the pointlike model follows the MAGIC PSF above $630 \mathrm{GeV}$. The thin and thick coloured solid lines represent the real and reconstructed (i.e., smeared by the PSF) signal fractions, respectively. The dotted lines represent the fraction of these signals contained in the reference background region. The vertical dashed lines correspond to the used optimum $\theta_{\text {cut }}$ values.

In practice, $\theta_{\text {cut }}$ is optimal on a relatively wide range. The same cut can be used for both the isobaric and extended models, which have very similar optimum $\theta_{\text {cut }}$ values. Since the MAGIC PSF is relatively stable above $630 \mathrm{GeV}$ (MAGIC Collaboration 2016b), we use the same $\theta_{\text {cut }}^{2}$ value for all energies for any given model. The resulting optimum cuts are presented in Table 1 and shown in Fig. 5. 
Table 1. Optimum $\theta_{\text {cut }}^{2}$ and contained flux fractions for different models.

\begin{tabular}{lccc}
\hline \hline Models & $\begin{array}{c}\theta_{\text {cut }}^{2} \\
{\left[\mathrm{deg}^{2}\right]}\end{array}$ & $\begin{array}{c}\text { flux }\left(<\theta_{\text {cut }}\right) \\
\text { ON (OFF) }\end{array}$ & $\begin{array}{c}\text { flux }\left(<0.15^{\circ}\right)^{a} \\
\text { real (recon.) }\end{array}$ \\
\hline Point-like & 0.0075 & $77 \%(0.0 \%)$ & $100 \%(96 \%)$ \\
Isobaric & 0.0325 & $41 \%(0.7 \%)$ & $39 \%(35 \%)$ \\
Semi-analytical & 0.0175 & $46 \%(0.2 \%)$ & $55 \%(50 \%)$ \\
Extended & 0.0325 & $26 \%(1.4 \%)$ & $22 \%(20 \%)$ \\
\hline
\end{tabular}

Notes. ${ }^{(a)}$ The flux within $0.15^{\circ}$ is used as reference for all models.

Concerning the CR-proton spectrum, we adopt for both the semi-analytical and extended models, the universal power-law momentum spectrum $p^{-\alpha}$, with $\alpha \approx 2.2$ for the energies of interest here, found in hydrodynamical simulations by Pinzke \& Pfrommer (2010). For the less predictive isobaric model, the spectral index is free to vary, and we assume a range of values, $2.1 \leq \alpha \leq 2.5$. In all cases, we also consider the EBL absorption, which results in a slightly softer observed $\gamma$-ray spectrum, $\Gamma=\alpha+0.13$, in the energy range $300 \mathrm{GeV}-10 \mathrm{TeV}$.

Table 2 presents the $95 \%$-c.l. upper limits of the integral flux between several energy thresholds $\left(E_{\mathrm{th}}\right)$ and $10 \mathrm{TeV}$ for different spatial models assuming a power-law spectral index $\Gamma=2.33$. The upper limits are converted to the corresponding flux contained within the reference radius $0.15^{\circ}$ and the cluster virial radius $^{8} R_{200} \simeq 1.4^{\circ}$ (Reiprich \& Böhringer 2002). The conversion factors depend on the surface brightness distributions, also considering the signal contained in the OFF region. The latter is particularly important for the extended CR model, which has a flatter surface brightness profile. For the point-like assumption, we estimated the background level from an average of five OFF regions, while for the $\mathrm{CR}$ models we only used a single region at $0.8^{\circ}$ from the cluster centre, as mentioned in Sect. 2 .

The effective area of MAGIC is relatively flat above $630 \mathrm{GeV}$, and the integral flux upper limits do not depend strongly on the assumed spectral shape. Therefore, the upper limits reported in Table 2 are valid, within $2 \%$, for an observed spectral index range of $2.1 \lesssim \Gamma \lesssim 2.6$, which corresponds to an intrinsic index range of about $2.0 \lesssim \alpha \lesssim 2.5$.

\section{Interpretation and discussion}

The flux upper limits reported in Sect. 4 allow to constrain the CR content in the Perseus cluster. We discuss the implications for the CR-to-thermal pressure for the three adopted models: isobaric, semi-analytical, and extended. Additionally, for the semi-analytical model of Pinzke \& Pfrommer (2010), our constraints can be translated into constraints on the CR acceleration efficiency at structure formation shocks. Finally, assuming that the Perseus radio mini-halo is induced by secondary electrons (i.e., assuming that the hadronic model for the radio mini-halo is valid), we discuss the expected minimum $\gamma$-ray flux and derive constraints on the cluster magnetic field.

\subsection{CR acceleration efficiency}

A major uncertainty in modelling CR physics in clusters of galaxies is the CR-acceleration efficiency, i.e., the percentage of the energy dissipated in structure formation shocks that goes into particle acceleration. We define the CR-acceleration efficiency

\footnotetext{
8 The cluster virial radius is defined here with respect to an average density that is 200 times the critical density of the Universe.
}

Table 2. Integral flux upper limits between $E_{\text {th }}$ and $10 \mathrm{TeV}$ assuming observed power-law spectrum with an index $\Gamma=2.33$.

\begin{tabular}{|c|c|c|c|c|c|c|}
\hline$E_{\text {th }}$ & Model & $N_{\mathrm{ON}^{a}}^{a}$ & $N_{\mathrm{OFF}^{a}}{ }^{a}$ & $\sigma_{\text {LiMa }}{ }^{b}$ & $F_{\mathrm{UL}}^{0.15^{\circ} c}$ & $F_{\mathrm{UL}}^{1.4^{\circ} \mathrm{C}}$ \\
\hline \multirow{4}{*}{$630 \mathrm{GeV}$} & point-like & 332 & 304.1 & 1.4 & 12.2 & 12.2 \\
\hline & isobaric & 1327 & 1256 & 1.4 & 24.8 & 65.5 \\
\hline & semi-analytic & 749 & 681 & 1.8 & 26.8 & 49.0 \\
\hline & extended & 1327 & 1256 & 1.4 & 25.6 & 124. \\
\hline \multirow{4}{*}{$1.0 \mathrm{TeV}$} & point-like & 159 & 157.5 & 0.1 & 3.84 & 3.84 \\
\hline & isobaric & 675 & 652 & 0.6 & 10.7 & 28.3 \\
\hline & semi-analytic & 369 & 352 & 0.6 & 9.77 & 17.9 \\
\hline & extended & 675 & 652 & 0.6 & 11.1 & 54.0 \\
\hline \multirow{4}{*}{$1.6 \mathrm{TeV}$} & point-like & 77 & 75.9 & 0.1 & 2.34 & 2.34 \\
\hline & isobaric & 321 & 317 & 0.2 & 5.09 & 13.5 \\
\hline & semi-analytic & 169 & 167 & 0.1 & 4.61 & 8.43 \\
\hline & extended & 321 & 317 & 0.2 & 5.26 & 25.6 \\
\hline \multirow{4}{*}{$2.5 \mathrm{TeV}$} & point-like & 38 & 37.4 & 0.1 & 1.57 & 1.57 \\
\hline & isobaric & 143 & 153 & -0.6 & 2.18 & 5.75 \\
\hline & semi-analytic & 77 & 81 & -0.3 & 2.30 & 4.21 \\
\hline & extended & 143 & 153 & -0.6 & 2.25 & 11.0 \\
\hline $2.5 \mathrm{TeV}$ & isobaric & - & - & - & 3.10 & 8.15 \\
\hline for zero & semi-analytic & - & - & - & 2.81 & 5.15 \\
\hline excess $^{d}$ & extended & - & - & - & 3.21 & 15.5 \\
\hline
\end{tabular}

Notes. ${ }^{(a)}$ Number of events in the signal $\left(N_{\mathrm{ON}}\right)$ and background $\left(N_{\mathrm{OFF}}\right)$ regions. ${ }^{(b)}$ Significance of the measured excess in standard deviations. (c) $95 \%$-c.l. flux upper limits in units of $10^{-14} \mathrm{~cm}^{-2} \mathrm{~s}^{-1}$ within a radius of $0.15^{\circ}$ and $1.4^{\circ}$ from the cluster centre. ${ }^{(d)}$ For negative measured excess, we provide conservative upper limits assuming zero excess.

as in Pinzke \& Pfrommer (2010) as the ratio of the energy density in freshly injected CRs to the total dissipated energy density in the downstream region of a shock. We emphasise that it is particularly difficult to put meaningful constraints on this fundamental quantity because of the lack of a general theory of particle acceleration. In fact, our current knowledge of the dependence of the acceleration efficiency with the shock Mach number is mainly phenomenological and numerical. Nevertheless, we assess the impact of our upper limits in the context of the state-of-the-art model by Pinzke \& Pfrommer (2010) and caution that our results are also subject to the limitations stated in that work.

Our semi-analytical model is based on predictions by Pinzke \& Pfrommer (2010), which are derived from hydrodynamical simulations of galaxy clusters assuming a maximum CR-proton acceleration efficiency $\zeta_{\mathrm{p} \text {,max }}=50 \%$. We parametrize $\zeta_{\mathrm{p} \text {,max }}$ with a flux multiplier $A_{\gamma}$, which is equal to 1 for $\zeta_{\mathrm{p}, \max }=50 \%$, and we assume a linear scaling between $A_{\gamma}$ and $\zeta_{\mathrm{p} \text {,max }}$ (Fermi-LAT Collaboration 2014). The hadronic-induced emission is proportional to $A_{\gamma}$, which provides the overall normalisation of the CR distribution. In the left-hand panel of Fig. 6, we show the integral $\gamma$-ray fluxes predicted by Pinzke \& Pfrommer (2010) for the Perseus cluster with $A_{\gamma}=1$ within $0.15^{\circ}$ from the centre and the highest fluxes allowed by our observational upper limits. The value of $A_{\gamma}$ is constrained to be $\leq 0.56$ when neglecting the EBL absorption and $A_{\gamma} \leq 0.75$ when including the EBL absorption from Domínguez et al. (2011). This corresponds to $\zeta_{\mathrm{p}, \max } \leq 28 \%$ and $\leq 37 \%$, respectively.

Compared to our previous paper (MAGIC Collaboration 2012a), we accumulated three times more data and derived upper limits that are significantly lower (except for the upper limit at $E>630 \mathrm{GeV}$ that suffers contamination from the NGC 1275 AGN). Our previous constraint, $A_{\gamma} \leq 0.8$, derived from the upper limit at $E>1 \mathrm{TeV}$, was underestimated because both the EBL absorption and the signal leakage in the OFF regions 

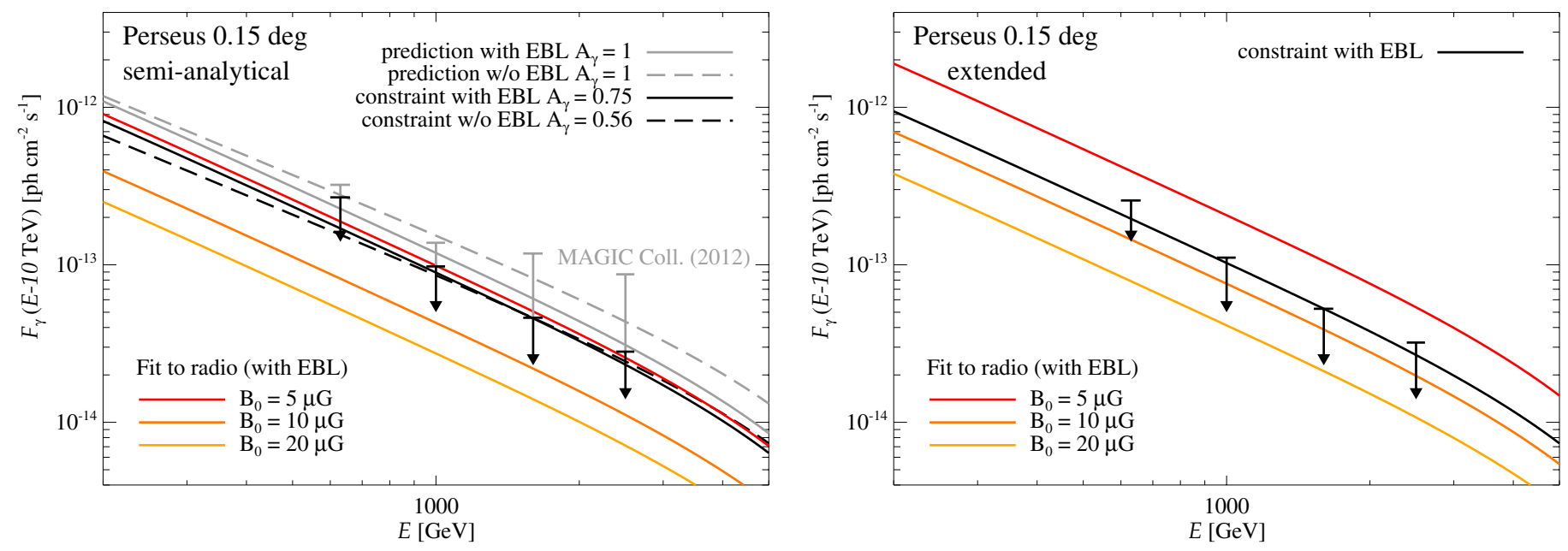

Fig. 6. Expected integral flux from the Perseus cluster core within $0.15^{\circ}$ and the associated $95 \%$-c.1. MAGIC upper limits for the semi-analytical model (Left) and the extended model (Right). For the semi-analytical model, we show the spectrum predicted by Pinzke \& Pfrommer (2010) $\left(A_{\gamma}=1\right.$, see text) and the one constrained by our upper limits for both cases with (solid lines) and without (dashed lines) EBL absorption. Arrows in light grey show our previous flux upper limits (MAGIC Collaboration 2012a). For both the semi-analytical and extended models, the EBLabsorbed spectra obtained by assuming that the Perseus radio mini-halo has a hadronic origin and fixing a central magnetic field value $B_{0}=5,10$, and $20 \mu \mathrm{G}$ are shown in colours. We used as reference the radio surface brightness radial profile of the Perseus mini-halo at $1.4 \mathrm{GHz}$ from Pedlar et al. (1990). Fluxes are integrated up to $10 \mathrm{TeV}$.

were neglected. These effects relax the constraint by about $25 \%$. As discussed in Sect. 4.1, this loss can be compensated by ICC emission. Moreover, the uncertainty on the EBL and ICC effects stays within the $30 \%$ systematics considered in deriving the upper limits. Here, considering the most conservative case, we can constrain $\zeta_{\text {p,max }} \leq 37 \%$, which is even slightly below our previous results. We note that Fermi-LAT observations suggest values of $\zeta_{\text {p,max }} \lesssim 15 \%$ for the case of the merging Coma cluster (Fermi-LAT Collaboration 2014; Zandanel \& Ando 2014), although all these results assume a negligible active CR transport, hence represent model-dependent upper limits.

We are able to constrain the CR acceleration efficiency only in the context of the hydrodynamical simulations of Pinzke \& Pfrommer (2010) because a full modelling of the formation history of a galaxy cluster and of the CR acceleration at the corresponding structure formation shocks is needed for this. Therefore, our constraint on $\zeta_{\text {p,max }}$ is strictly valid only in this context, which assumes in particular i) no CR transport relative to the plasma rest frame; and ii) a simplified model for $\mathrm{CR}$ acceleration in which $\zeta_{\mathrm{p} \text {,max }}$ rises steeply for shocks with weak Mach numbers $M$ and already saturates at $M \gtrsim 3$ (Enßlin et al. 2007). We assess the first assumption by adopting the extended model, shown in the right-hand panel of Fig. 6, which was constructed independently of $\zeta_{\mathrm{p} \text {, max }}$ to match the radio emission from the Perseus mini-halo (Zandanel et al. 2014; see Sect. 5.3). Concerning the second assumption, nonlinear diffusive shock acceleration models (Kang \& Ryu 2011, 2013) predict a slower rise in $\zeta_{\mathrm{p}, \max }$ with increasing $M$, which then saturates at higher Mach numbers with respect to Enßlin et al. (2007). However, Cherenkov telescopes probe the highmomentum part of the CR-proton spectrum, which is generated by intermediate-strength shocks where the acceleration efficiency is close to saturation for all models.

Our result on the maximum CR acceleration efficiency is a useful proxy for calibrating the total expected emission in the context of the adopted semi-analytical model, but as stressed above, it should be taken with a grain of salt as general constraint in the context of CR acceleration at shocks. Additionally, the latest supernova remnant observations tend to suggest values
Table 3. Constraints on the volume-averaged CR-to-thermal pressure ratio within $R_{200}$ for different CR models.

\begin{tabular}{ccrr}
\hline \hline Model & $\alpha$ & $\left\langle X_{\mathrm{CR}, \max }^{\mathrm{no}-\mathrm{EBL}}\right\rangle[\%]$ & $\left\langle X_{\mathrm{CR}, \max }\right\rangle[\%]$ \\
\hline Isobaric & 2.1 & 0.5 & 0.7 \\
& 2.2 & 0.8 & 1.1 \\
& 2.3 & 1.7 & 2.3 \\
& 2.5 & 11.4 & 15.2 \\
Semi-analytical & 2.2 & 1.5 & 2.0 \\
Extended & 2.2 & 14.2 & 19.2 \\
\hline
\end{tabular}

Notes. Upper limits on the volume-averaged $X_{\mathrm{CR}}$ obtained within $R_{200}$ derived from the integral-flux upper limit in the energy range $1.6-10 \mathrm{TeV} .\left\langle X_{\mathrm{CR} \text { max }}^{\text {no-EB }}\right\rangle$ is the constraint obtained neglecting the EBL absorption, given for comparison with previous results. $\left\langle X_{\mathrm{CR}, \max }\right\rangle$ includes the EBL-absorption correction.

below 27\% (Helder et al. 2013), with a commonly accepted value around $10 \%$ to be able to explain Galactic CRs (e.g., Morlino \& Caprioli 2012). The acceleration conditions at structure formation shock may be different from those at supernova remnants, complicating any direct comparison.

\subsection{CR-to-thermal pressure}

Depending on the spectral and spatial distribution of the CR protons, the MAGIC upper limits from Table 2 can be translated into constraints on the cluster CR population. In Table 3, we report the constraints on the CR-to-thermal pressure $X_{\mathrm{CR}}=P_{\mathrm{CR}} / P_{\mathrm{th}}$ obtained with the isobaric model for different $\mathrm{CR}$ spectral indexes, $2.1 \leq \alpha \leq 2.5$, using the most constraining integral flux upper limit, i.e., the one above $1.6 \mathrm{TeV}$. The CR-to-thermal pressure must be below about $1 \%$ for $\alpha \leq 2.2$, below about $2 \%$ for $\alpha=2.3$, and below $15 \%$ for $\alpha=2.5$. Including the EBL absorption effectively causes a worsening of the derived constraints of about $25 \%$. Therefore, the improvement of the constraints is modest, but the results are more robust with respect to MAGIC Collaboration (2012a). 
M. L. Ahnen et al.: Search of diffuse $\gamma$-ray emissions from cosmic rays in the Perseus cluster

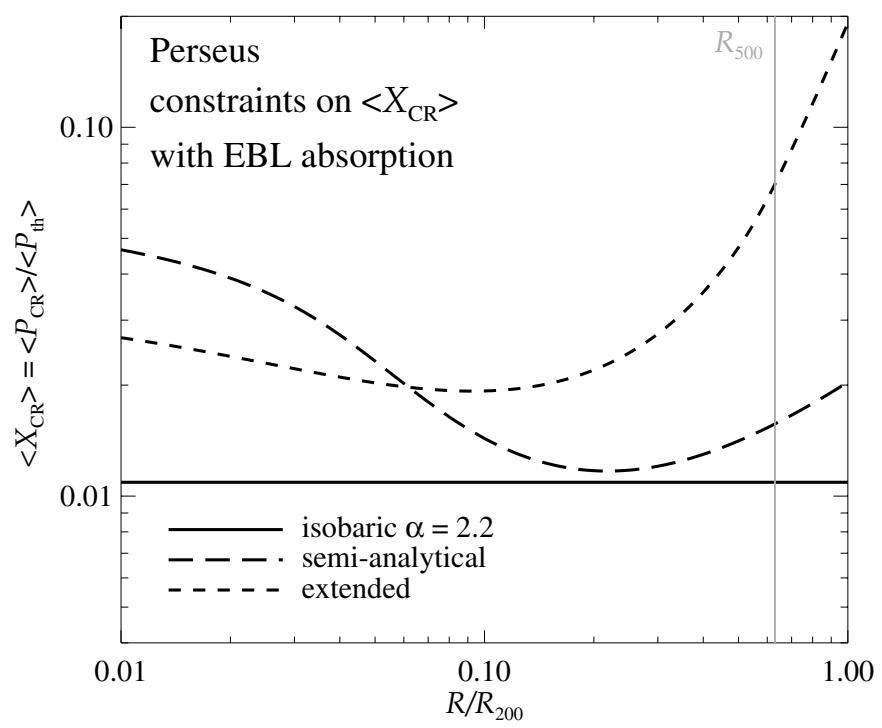

Fig. 7. Volume-averaged $X_{\mathrm{CR}}$ within a given radius $R$ as constrained by the upper limits presented in this work for the semi-analytical, extended, and isobaric models, the last one with $\alpha=2.2$ matching the effective spectral index of the other models in the energies of interest here. We recall that in the isobaric model, $X_{\mathrm{CR}}(r)$ is constant by construction. The vertical grey line represents the radius $R_{500}$, expressed with respect to an average density that is 500 times the critical density of the Universe, for an easier comparison with cosmological studies.

The constraints on the volume-averaged CR-to-thermal pressure profiles for the semi-analytical and extended models are below $1.4 \%$ and $1.9 \%$ within $0.15^{\circ}\left(\approx 0.11 \times R_{200}\right)$, respectively, as shown in Fig. 7 and Table 3 . When considering the full galaxy cluster volume up to $R_{200},\left\langle X_{\mathrm{CR}}\right\rangle$ is below $2 \%$ for the semianalytical model, but significantly less constrained, below $19 \%$, for the extended model. This last weak constraint is expected because the CR distribution is significantly flatter in the extended model, leading to high $\left\langle X_{\mathrm{CR}}\right\rangle$ values in the cluster outskirts characterised by lower thermal pressure.

While in both simulations and analytical models, the CR distribution in galaxy clusters is assumed to roughly scale with the thermal gas, the real CR distribution is unknown and could be significantly flatter if CRs propagate out of the cluster core (Enßlin et al. 2011; Wiener et al. 2013; Zandanel et al. 2014). Indeed, phenomenological evidence from observations of giant radio halos indicate that the $\mathrm{CR}$ distribution appears to be flatter than the ICM distribution independently of the generation mechanism of the observed radio emission (Brunetti et al. 2012; Zandanel et al. 2014; Pinzke et al. 2015). This should be kept in mind when using galaxy clusters for cosmological purposes because, depending on the exact amount of CR protons, this can induce a bias in the estimation of cluster masses. While this effect is limited to a few percent for the standard assumptions (isobaric and semi-analytical models), a flat CR distribution could generate up to a $20 \%$ bias (e.g., in the specific case considered here) in hydrostatic mass estimates, which are potentially relevant in the current era of precision cosmology.

\subsection{Radio mini-halo and magnetic fields}

As mentioned in Sect. 1, the Perseus cluster hosts the brightest radio mini-halo known to date (Pedlar et al. 1990; Sijbring 1993; Gitti et al. 2002). Assuming that the observed radio emission has a hadronic origin, i.e., is generated by secondary electrons produced in hadronic interactions between the CR and ICM protons, the pion-decay $\gamma$-ray emission is directly linked to the radio signal. Since the intensity of the synchrotron radio emission depends on the amount of secondary electrons, proportional to the hadronically-induced $\gamma$ rays, and the local magnetic field, our $\gamma$-ray upper limits can be turned into lower limits on the cluster magnetic field (MAGIC Collaboration 2012a).

The $\gamma$-ray and synchrotron luminosities can be expressed as (adapted from Pfrommer 2008)

$$
\begin{aligned}
& L_{\gamma}=C_{\gamma} \int \mathrm{d} V n_{\mathrm{CR}} n_{\mathrm{ICM}}, \\
& L_{v}=C_{v} \int \mathrm{d} V n_{\mathrm{CR}} n_{\mathrm{ICM}} \frac{\varepsilon_{B}^{\left(\alpha_{\nu}+1\right) / 2}}{\varepsilon_{\mathrm{CMB}}+\varepsilon_{\mathrm{SD}}+\varepsilon_{B}},
\end{aligned}
$$

where $\alpha_{v}=\alpha / 2$ is the synchrotron spectral index $\left(S_{v} \propto v^{-\alpha_{v}}\right)$, $n_{\mathrm{CR}}$ and $n_{\mathrm{ICM}}$ are the CR and ICM densities, $C_{\gamma}$ and $C_{v}$ are constants that depend on the physics of the hadronic interactions, and $\varepsilon_{B}, \varepsilon_{\mathrm{CMB}}$ and $\varepsilon_{\mathrm{SD}}$ denote the energy density of the magnetic field $\left(=B^{2} / 8 \pi\right)$, the $\mathrm{CMB}$, and the star-and-dust light in the cluster, respectively. The magnetic field in galaxy clusters can be parametrised as

$B(r)=B_{0}\left(\frac{n_{\mathrm{e}}(r)}{n_{\mathrm{e}}(0)}\right)^{\alpha_{B}}$,

where $B_{0}$ is the central magnetic field, $n_{\mathrm{e}}$ the ICM electron density, and $\alpha_{B}$ a parameter describing the radial decline of the magnetic field. Such a parametrisation is favoured both by FR measurements in clusters and by hydrodynamical simulations (e.g., Dubois \& Teyssier 2008; Bonafede et al. 2010; Kuchar \& Enßlin 2011).

Our radio synchrotron modelling includes energy losses due to IC scattering of ambient photons. We consider the CMB, as well as the light from stars and dust (SD) in the cluster, $\varepsilon_{\mathrm{SD}}$, according to the model of Pinzke et al. (2011), which has the advantage of including the average contribution from the intracluster light obtained from a stacking of cluster observations. We stress that the SD energy density dominates both the CMB and magnetic field energy densities in the very centre of the cluster, typically $\leq 0.03 \times R_{200}$, for low values of the magnetic field, i.e., $B_{0} \lesssim 5 \mu \mathrm{G}$. Therefore, for the case of Perseus, where the observed radio emission arises from within about $0.1 \times R_{200}$ (see next section), including this term in the synchrotron losses can significantly affect the modelling.

In Fig. 6, we show the EBL-corrected $\gamma$-ray emission within $0.15^{\circ}$ from the centre for both the semi-analytical and the extended models, corresponding to the parameters, for which secondary electrons reproduce the observed radio surface brightness radial profile of the Perseus mini-halo at $1.4 \mathrm{GHz}$ (Pedlar et al. 1990) $)^{9}$. We adopted three different values of the central magnetic field $B_{0}=5,10$, and $20 \mu \mathrm{G}$ with $\alpha_{B}=0.3$ for the semi-analytical model and $\alpha_{B}=0.5$ for the extended model as best-fit values. Values of $\alpha_{B} \geq 0.5$ are theoretically preferred, for example from simulations of gas sloshing in cool-core clusters (ZuHone et al. 2010, 2011), and the low value of $\alpha_{B}$ required for the semi-analytical model is due to the centrally peaked CR profile in the Pinzke \& Pfrommer (2010) simulations, in which

9 We take as reference here the radio surface brightness radial profile from Pedlar et al. (1990) at $1.4 \mathrm{GHz}$ rather than the one at $327 \mathrm{MHz}$ from Gitti et al. (2002), as the latter may be affected by residual point-source contamination as pointed out in Sijbring (1993) where the $327 \mathrm{MHz}$ data was taken. 
AGN feedback was not accounted for, causing enhanced radiative cooling.

At parity of $B_{0}$, the extended model always shows a higher $\gamma$-ray emission than the semi-analytical model because of the flatter radial profile. In fact, while our $\gamma$-ray upper limits imply $B_{0} \gtrsim 5.5 \mu \mathrm{G}$ for the semi-analytical model, for the extended model we obtain $B_{0} \gtrsim 8 \mu \mathrm{G}$ (for the choices of $\alpha_{B}$ as above). The FR measurements of cluster central magnetic field strengths range from $5 \mu \mathrm{G}$ for merging clusters (Bonafede et al. 2010, 2013) to about $40 \mu \mathrm{G}$ for cool-core objects (Kuchar \& Enßlin 2011). For the Perseus cluster, FR measurements are only available on very small scales, i.e., a few tens of pc, and this suggests magnetic field strengths of about $25 \mu \mathrm{G}$ (Taylor et al. 2006). There are, however, large uncertainties related to this measurement, and we refer the reader to more extensive discussions on this topic in our previous publications on the Perseus cluster (MAGIC Collaboration 2010b, 2012a). In conclusion, when assuming the radio mini-halo in Perseus has a hadronic origin, the lower limits obtained on the magnetic field strength in the Perseus cluster using our $\gamma$-ray flux upper limits are consistent with FR measurements.

\subsection{Minimum $\gamma$-ray fluxes}

For clusters that host diffuse radio emission, such as the radio mini-halo in Perseus, we can estimate a theoretical minimum $\gamma$-ray flux in the hadronic scenario, which assumes that the observed radio emission has a secondary origin. The idea is that if the magnetic field is strong enough in all the radioemitting region, i.e., $\epsilon_{B} \gg \epsilon_{\mathrm{CMB}}+\epsilon_{\mathrm{SD}}$, a stationary distribution of CR electrons loses all its energy to synchrotron radiation (Pfrommer et al. 2008; Pfrommer 2008; MAGIC Collaboration $2010 b, 2012 a)$. In this case, the ratio of $\gamma$-ray-to-synchrotron luminosity, $L_{\gamma} / L_{\gamma}$, becomes independent of the spatial distribution of CRs, of the ICM, and of the magnetic field, if the observed synchrotron spectral index is $\alpha_{v}=\alpha / 2 \approx 1$, as can be seen from Eqs. (2) and (3). Therefore, a minimum theoretical $\gamma$-ray flux, $F_{\gamma, \min }=L_{\gamma} / 4 \pi D_{\text {lum }}^{2}$, can be derived as

$F_{\gamma, \min } \approx \frac{C_{\gamma}}{C_{v}} \frac{L_{v}}{4 \pi D_{\mathrm{lum}}^{2}}$.

A lower magnetic field value would require a higher secondary $\mathrm{CR}$ electron density, hence a higher CR proton density, to reproduce the observed radio synchrotron luminosity and would therefore result in a higher $\gamma$-ray flux. This is why we can consider the above $\gamma$-ray flux as a theoretical minimum in the hadronic scenario.

The measured spectral index of the Perseus radio mini-halo ranges from $\alpha_{v}=1.1$ to $\alpha_{v}=1.4$ (Sijbring 1993; Gitti et al. 2002). Assuming, for example, $B_{0}=20 \mu \mathrm{G}$, the CR protons responsible for the GHz-synchrotron-emitting secondary electrons have an energy of $\sim 20 \mathrm{GeV}$, which is about 400 times lower than the energy of the $\mathrm{CR}$ protons of $\sim 8 \mathrm{TeV}$ responsible for the $\mathrm{TeV} \gamma$-ray emission. Here, again, we consider spectral indexes of $2.1 \leq \alpha \leq 2.5$, which are consistent with the concavity in the CR spectrum that connects the low-energy CR population around $\mathrm{GeV}$ energies (characterised by $\alpha \simeq 2.5$ ) with the harder $\mathrm{CR}$ population at TeV energies $(\alpha \simeq 2.2$; Pinzke \& Pfrommer 2010; see also the discussion in MAGIC Collaboration 2012a). While Eq. (5) is only approximately valid in the adopted range of spectral indexes, we estimate that the deviations in $L_{\gamma} / L_{v}$ from the exact equation with $\alpha=2$ are within $10 \%$.

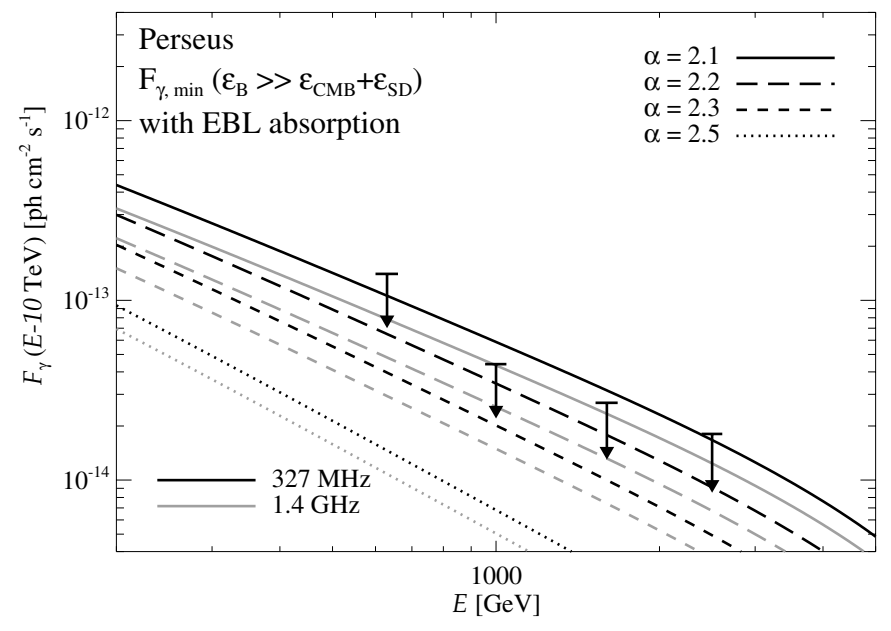

Fig. 8. Minimum $\gamma$-ray flux in the hadronic scenario obtained assuming that the Perseus radio mini-halo has a secondary origin and that $\epsilon_{B} \gg \epsilon_{\mathrm{CMB}}+\epsilon_{\mathrm{SD}}$. We show the EBL-corrected fluxes for different spectral indexes $\alpha$, adopting as reference the total synchrotron luminosity measured both at $327 \mathrm{MHz}$ (Gitti et al. 2002) and at $1.4 \mathrm{GHz}$ (Pedlar et al. 1990). We compare these with our 95\%-c.l. point-like upper limits appropriately scaled for the radio surface brightness shape, i.e., multiplied by a factor of 1.15 with respect to what is reported in Table 2 . The flux is integrated up to $10 \mathrm{TeV}$.

We take as reference the total radio luminosity of the minihalo measured at $327 \mathrm{MHz}$ by Sijbring (1993) of $L_{327 \mathrm{MHz}}=$ $17.57 \times 10^{-23} \mathrm{erg} \mathrm{s}^{-1} \mathrm{~Hz}^{-1} \mathrm{~cm}^{-2}$, and at $1.4 \mathrm{GHz}$ by Pedlar et al. (1990) of $L_{1.4 \mathrm{GHz}}=3.04 \times 10^{-23} \mathrm{erg} \mathrm{s}^{-1} \mathrm{~Hz}^{-1} \mathrm{~cm}^{-2}$. The measured maximum emission radius of the Perseus radio mini-halo ranges from about $100 \mathrm{kpc}$ at $1.4 \mathrm{GHz}$ (Pedlar et al. 1990) to about $200 \mathrm{kpc}$ at $327 \mathrm{MHz}$ (Sijbring 1993; Gitti et al. 2002), which correspond to $0.075^{\circ}\left(\sim 0.05 \times R_{200}\right)$ and $0.15^{\circ}(\sim 0.1 \times$ $R_{200}$ ), respectively. The shape of the radio surface brightnesses at $327 \mathrm{MHz}$ and at $1.4 \mathrm{GHz}$ is more compact than the MAGIC PSF. The minimum $\gamma$-ray flux of the hadronic scenario is therefore close to a point-like source for MAGIC. About $67 \%$ of the signal would be within the $\theta_{\text {cut }}$ used for the point-like assumption instead of $77 \%$ (see Table 1). An appropriate correction factor of 1.15 must be applied to the point-like upper limits presented in Table 2 to correct for the expected emission not being perfectly point-like. In doing so, we implicitly assumed that no $\gamma$-ray emission is coming from beyond the observed extension in radio frequencies (see MAGIC Collaboration 2012a, for a discussion).

Figure 8 shows the EBL-corrected minimum $\gamma$-ray emission derived as described above for $2.1 \leq \alpha \leq 2.5$, and adopting both $L_{327 \mathrm{MHz}}$ and $L_{1.4 \mathrm{GHz}}$ as synchrotron luminosity. We compare them with the properly scaled $(\times 1.15)$ point-like flux upper limits. The most striking result is that the minimum $\gamma$-ray flux for $\alpha=2.1$ conflicts with our upper limits. This implies that, if $\alpha \leq 2.1$, the observed diffuse radio emission in Perseus cannot be uniquely hadronic in origin, independently of the magnetic field strength in the cluster. For the softer spectral indexes considered here, the current $\gamma$-ray upper limits cannot exclude the hadronic origin of the diffuse radio emission in Perseus. The other spectral index cases should be in the reach of future ground-based $\gamma$-ray observations with the Cherenkov Telescope Array (CTA; e.g., Doro et al. 2013) with one order of magnitude better sensitivity. 


\section{Conclusions}

Clusters of galaxies are expected not only to contain $\gamma$-ray-bright AGNs, but also to host diffuse $\gamma$-ray emission due to neutral pion decays, induced by CR-ICM hadronic interactions. Indeed, $\mathrm{CR}$ protons should be accelerated in clusters by structure formation shocks and injected by outflows from galaxies and AGNs, and then should hadronically interact with the ICM protons, generating pions. The most promising galaxy cluster to search for such diffuse $\gamma$-ray emission is the Perseus cluster, which has been intensively observed with the MAGIC telescopes since 2008. These observations resulted in the detection of two $\gamma$-raybright AGNs in the central galaxy NGC 1275 and in the peculiar galaxy IC 310, both already reported in previous MAGIC publications. Here, we report the search of diffuse $\gamma$-ray emission, using $253 \mathrm{hr}$ of MAGIC observation in stereoscopic mode, accumulated from 2009 to 2014.

The region of the hard point-like source IC 310, located $0.6^{\circ}$ from the cluster centre, can be easily excluded from our search for extended emission as this latter is expected to be centred on the cluster core. The emission from the NGC 1275 AGN, however, overlies the searched signal region. We derived the most precise NGC 1275 spectrum in the range $90 \mathrm{GeV}-$ $1.2 \mathrm{TeV}$ ever done. It is described well by a simple power law $f_{0}(E / 200 \mathrm{GeV})^{-\Gamma}$ with a very steep photon index $\Gamma=3.6 \pm$ $0.2_{\text {stat }} \pm 0.2_{\text {syst }}$ and a differential-flux normalisation at $200 \mathrm{GeV}$ of $f_{0}=\left(2.1 \pm 0.2_{\text {stat }} \pm 0.3_{\text {syst }}\right) \times 10^{-11} \mathrm{~cm}^{-2} \mathrm{~s}^{-1} \mathrm{TeV}^{-1}$, in agreement with previous measurements. No signal is detected above approximately $1 \mathrm{TeV}$. Since the CR-induced emission is expected to have a harder spectrum, we preferred the high energies for our search. No other point-like emission is detected in the cluster, in particular from the radio galaxy NGC 1265, for which we derived a $95 \%$-c.l. flux upper limit above $250 \mathrm{GeV}$ of $5.6 \times 10^{-13} \mathrm{~cm}^{-2} \mathrm{~s}^{-1}$.

To bracket the uncertainty on the CR spatial and spectral distribution in Perseus, we considered three different models. First, the isobaric model, in which the CR-to-thermal pressure is constant and the CR-spectrum index $\alpha$ ranges between 2.1 and 2.5. Second, the semi-analytical model of Pinzke \& Pfrommer (2010) was derived from hydrodynamical simulations of clusters, for which the CR spectrum follows a universal spectrum with $\alpha=2.2$ at the energies of interest here. Finally, the extended hadronic model of Zandanel et al. (2014), in which CRs propagate out of the cluster core and generate a significantly flatter radial distribution with respect to the previous two models. In this last model, the CR spectrum is the same as in the semi-analytical model. In this work we have not considered any softening of the CR-proton spectrum induced by possible CR propagation effects (e.g., Wiener et al. 2013).

We optimised our analysis for the different considered CR models. No diffuse $\gamma$-ray emission or large-scale structures were detected in Perseus. We derived 95\%-c.l. integral flux upper limits, in different energy ranges and compared to the signal expected from the models over the same range. For the first time, we included the effect of the EBL absorption, which reduces the $\gamma$-ray flux above $1 \mathrm{TeV}$ coming from Perseus by $\sim 20 \%$. We discuss the fate of the produced electron pairs, including ICC and possibility of plasma instabilities driven by the anisotropy of the pair beams. We concluded that the absorbed $\gamma$ rays with $E>$ $30 \mathrm{TeV}$ are very likely reprocessed via ICC to our energy range of interest. In the most optimistic scenario (EGMF $<10^{-14} \mathrm{G}$ ), the ICC $\gamma$ rays could fully compensate for the effect of the EBL absorption in $1-10 \mathrm{TeV}$ range. The strongest constraints on the
CR models come from the $1.6-10 \mathrm{TeV}$ integral-flux upper limits of about $5 \times 10^{-14} \mathrm{~cm}^{-2} \mathrm{~s}^{-1}$ in a central region of $0.15^{\circ}$ radius.

The comparison with the semi-analytical model sets a constraint on the maximum CR-proton acceleration efficiency, $\zeta_{\mathrm{p}, \max }$, as defined in Pinzke \& Pfrommer (2010). The derived constraint, $\zeta_{\mathrm{p}, \max } \leq 37 \%$, is not much below our previous result obtained with $85 \mathrm{hr}$ of data because the EBL absorption was not taken into account in that early work. Our new study is, therefore, more conservative and more robust. We stress, however, that this constraint is only valid in the context of the Pinzke \& Pfrommer (2010) model.

More model-independent constraints were set on the CR-tothermal pressure ratio in the cluster. In the context of the isobaric model, $X_{\mathrm{CR}}$ must be $\lesssim 1 \%$ for $\alpha \leq 2.2$, $\lesssim 2 \%$ for $\alpha=2.3$, and $\lesssim 15 \%$ for $\alpha=2.5$. When considering the semi-analytical model, $\left\langle X_{\mathrm{CR}}\right\rangle$ within $R_{200}$ is constrained to be $\lesssim 2 \%$. In the $e x$ tended model, $\left\langle X_{\mathrm{CR}}\right\rangle \lesssim 2 \%$ within $0.15^{\circ}$, but only $\lesssim 20 \%$ within $R_{200}$ because the volume-averaged pressure ratio builds up to significant values in the cluster outskirts where the ICM pressure drops. The actual CR distribution in clusters is unknown, and if it deviates significantly from the ICM distribution, as for example, in the extended model, it could induce a bias on the estimates of the cluster hydrostatic mass, where its contribution is usually neglected, at a level that is potentially important in the current era of precision cosmology.

The Perseus cool-core cluster hosts the brightest known radio mini-halo. Assuming that this diffuse radio emission is generated by synchrotron radiation of secondary electrons from CR hadronic interactions with the ICM, we can turn our $\gamma$-ray flux upper limits into lower limits on the central magnetic field strength in the cluster. For the first time, we included in our modelling energy losses due to IC scattering of ambient photons from stars and dust in the cluster, in addition to the commonly considered CMB. We found $B_{0} \gtrsim 5.5 \mu \mathrm{G}$ and $B_{0} \gtrsim 8 \mu \mathrm{G}$ for the semianalytical and extended models, respectively. These constraints are consistent with FR measurements in clusters. Additionally, assuming that CR electrons lose all their energy by synchrotron emission in the radio emitting region $\left(\epsilon_{B} \gg \epsilon_{\mathrm{CMB}}+\epsilon_{\mathrm{SD}}\right)$, the derived $\gamma$-ray flux becomes independent of the CR, magnetic field, and ICM distributions. This represents a theoretical flux lower limit in the hadronic scenario because lower magnetic fields would imply a higher $\gamma$-ray emission. With this approach, we found that for $\alpha \leq 2.1$, the hadronic interpretation of the Perseus radio mini-halo is in conflict with our upper limits. For more realistic $\alpha>2.1$, the minimum $\gamma$-ray flux is several times below our upper limits, hence out of reach with MAGIC.

The large amount of data presented in this work, about $250 \mathrm{~h}$ of observations, implies that it would be difficult to significantly improve upon our constraints with the current generation of Cherenkov telescopes. Therefore, this five-year-long campaign represents one of the reference results, together with the FermiLAT observations, for the cluster physics in the $\gamma$-ray energy regime until the planned CTA observatory becomes operational in a few years from now.

Acknowledgements. The MAGIC collaboration would like to thank the Instituto de Astrofísica de Canarias for the excellent working conditions at the Observatorio del Roque de los Muchachos in La Palma. The financial support of the German BMBF and MPG, the Italian INFN and INAF, the Swiss National Fund SNF, the ERDF under the Spanish MINECO (FPA2012-39502), and the Japanese JSPS and MEXT is gratefully acknowledged. This work was also supported by the Centro de Excelencia Severo Ochoa SEV-20120234, CPAN CSD2007-00042, and MultiDark CSD2009-00064 projects of the Spanish Consolider-Ingenio 2010 programme, by grant 268740 of the Academy of Finland, by the Croatian Science Foundation (HrZZ) Project 09/176 
and the University of Rijeka Project 13.12.1.3.02, by the DFG Collaborative Research Centers SFB823/C4 and SFB876/C3, and by the Polish MNiSzW grant 745/N-HESS-MAGIC/2010/0. F. Zandanel acknowledges the support of the Netherlands Organisation for Scientific Research (NWO) through a Veni grant. C. Pfrommer gratefully acknowledges support of the Klaus Tschira Foundation.

\section{References}

Ajello, M., Rebusco, P., Cappelluti, N., et al. 2009, ApJ, 690, 367 Ajello, M., Rebusco, P., Cappelluti, N., et al. 2010, ApJ, 725, 1688 Ando, S., \& Nagai, D. 2012, J. Cosmol. Astropart. Phys., 7, 17 Bartels, R., Zandanel, F., \& Ando, S. 2015, A\&A, 582, A20 Berezinsky, V. S., Blasi, P., \& Ptuskin, V. S. 1997, ApJ, 487, 529 Blasi, P., \& Colafrancesco, S. 1999, Astropart. Phys., 12, 169 Blasi, P., Gabici, S., \& Brunetti, G. 2007, Inter. J. Mod. Phys. A, 22, 681 Bonafede, A., Feretti, L., Murgia, M., et al. 2010, A\&A, 513, A30 Bonafede, A., Vazza, F., Brüggen, M., et al. 2013, MNRAS, 433, 3208 Bravi, L., Gitti, M., \& Brunetti, G. 2016, MNRAS, 455, L41 Broderick, A. E., Chang, P., \& Pfrommer, C. 2012, ApJ, 752, 22 Broderick, A. E., Pfrommer, C., Puchwein, E., \& Chang, P. 2014a, ApJ, 790, 137 Broderick, A. E., Pfrommer, C., Puchwein, E., Chang, P., \& Smith, K. M. 2014b, ApJ, 796, 12

Brown, S., \& Rudnick, L. 2011, MNRAS, 412, 2

Brunetti, G., \& Blasi, P. 2005, MNRAS, 363, 1173

Brunetti, G., \& Jones, T. W. 2014, Int. J. Mod. Phys. D, 23, 30007

Brunetti, G., \& Lazarian, A. 2007, MNRAS, 378, 245

Brunetti, G., \& Lazarian, A. 2011, MNRAS, 410, 127

Brunetti, G., Setti, G., Feretti, L., \& Giovannini, G. 2001, MNRAS, 320, 365

Brunetti, G., Blasi, P., Cassano, R., \& Gabici, S. 2004, MNRAS, 350, 1174

Brunetti, G., Blasi, P., Reimer, O., et al. 2012, MNRAS, 426, 956

Carilli, C. L., \& Taylor, G. B. 2002, ARA\&A, 40, 319

Cassano, R., \& Brunetti, G. 2005, MNRAS, 357, 1313

Chang, P., Broderick, A. E., \& Pfrommer, C. 2012, ApJ, 752, 23

Chang, P., Broderick, A. E., Pfrommer, C., et al. 2014, ApJ, 797, 110

Churazov, E., Forman, W., Jones, C., \& Böhringer, H. 2003, ApJ, 590, 225

Clarke, T. E., Kronberg, P. P., \& Böhringer, H. 2001, ApJ, 547, L111

Deiss, B. M., Reich, W., Lesch, H., \& Wielebinski, R. 1997, A\&A, 321, 55

Dennison, B. 1980, ApJ, 239, L93

Dermer, C. D., Cavadini, M., Razzaque, S., et al. 2011, ApJ, 733, L21

Dolag, K., \& Enßlin, T. A. 2000, A\&A, 362, 151

Dolag, K., Kachelriess, M., Ostapchenko, S., \& Tomàs, R. 2011, ApJ, 727, L4

Domainko, W., Nedbal, D., Hinton, J. A., \& Martineau-Huynh, O. 2009, Int. J. Mod. Phys. D, 18, 1627

Domínguez, A., Primack, J. R., Rosario, D. J., et al. 2011, MNRAS, 410, 2556

Donnert, J., Dolag, K., Brunetti, G., Cassano, R., \& Bonafede, A. 2010a, MNRAS, 401, 47

Donnert, J., Dolag, K., Cassano, R., \& Brunetti, G. 2010b, MNRAS, 407, 1565

Donnert, J., Dolag, K., Brunetti, G., \& Cassano, R. 2013, MNRAS, 429, 3564

Doro, M., Conrad, J., Emmanoulopoulos, D., et al. 2013, Astropart. Phys., 43, 189

Dubois, Y., \& Teyssier, R. 2008, A\&A, 482, L13

Enßlin, T. A., Biermann, P. L., Kronberg, P. P., \& Wu, X.-P. 1997, ApJ, 477, 560

Enßlin, T. A., Pfrommer, C., Springel, V., \& Jubelgas, M. 2007, A\&A, 473, 41

Enßlin, T., Pfrommer, C., Miniati, F., \& Subramanian, K. 2011, A\&A, 527, A99

Feretti, L., Giovannini, G., Govoni, F., \& Murgia, M. 2012, A\&ARv, 20, 54

Fermi-LAT Collaboration 2009, ApJ, 699, 31

Fermi-LAT Collaboration 2010a, J. Cosmol. Astropart. Phys., 5, 25

Fermi-LAT Collaboration 2010b, ApJ, 717, L71

Fermi-LAT Collaboration 2014, ApJ, 787, 18

Fermi-LAT Collaboration 2015, ApJ, 812, 159

Fermi-LAT Collaboration 2016, ApJ, 819, 149

Fujita, Y., \& Ohira, Y. 2012, ApJ, 746, 53

Fujita, Y., Takizawa, M., \& Sarazin, C. L. 2003, ApJ, 584, 190

Gabici, S., \& Blasi, P. 2003, Astropart. Phys., 19, 679

Galante, N., Acciari, V. A., Aliu, E., et al. 2009, ArXiv e-prints [arXiv:0907.5000]

Gastaldello, F., Wik, D. R., Molendi, S., et al. 2015, ApJ, 800, 139

Gitti, M., Brunetti, G., \& Setti, G. 2002, A\&A, 386, 456

Griffin, R. D., Dai, X., \& Kochanek, C. S. 2014, ApJ, 795, L21

Han, J., Frenk, C. S., Eke, V. R., et al. 2012, MNRAS, 427, 1651

Helder, E. A., Vink, J., Bamba, A., et al. 2013, MNRAS, 435, 910

HESS Collaboration 2009a, A\&A, 502, 437

HESS Collaboration 2009b, A\&A, 495, 27

HESS Collaboration 2012, A\&A, 545, A103

Huber, B., Tchernin, C., Eckert, D., et al. 2013, A\&A, 560, A64

Jeltema, T. E., \& Profumo, S. 2011, ApJ, 728, 53
Kang, H., \& Ryu, D. 2011, ApJ, 734, 18

Kang, H., \& Ryu, D. 2013, ApJ, 764, 95

Keshet, U., \& Loeb, A. 2010, ApJ, 722, 737

Kim, K.-T., Kronberg, P. P., \& Tribble, P. C. 1991, ApJ, 379, 80

Kitayama, T., Bautz, M., Markevitch, M., et al. 2014, ArXiv e-prints [arXiv: 1412.1176]

Kiuchi, R., Mori, M., Bicknell, G. V., et al. 2009, ApJ, 704, 240

Kuchar, P., \& Enßlin, T. A. 2011, A\&A, 529, A13

Kushnir, D., Katz, B., \& Waxman, E. 2009, J. Cosmol. Astropart. Phys., 9, 24

MAGIC Collaboration 2008, Nucl. Instr. Methods A, 588, 424

MAGIC Collaboration 2010a, ApJ, 723, L207

MAGIC Collaboration 2010b, ApJ, 710, 634

MAGIC Collaboration 2012a, A\&A, 541, A99

MAGIC Collaboration 2012b, A\&A, 539, L2

MAGIC Collaboration 2014a, A\&A, 564, A5

MAGIC Collaboration 2014b, A\&A, 563, A91

MAGIC Collaboration 2016a, Astropart. Phys., 72, 61

MAGIC Collaboration 2016b, Astropart. Phys., 72, 76

Miniati, F. 2003, MNRAS, 342, 1009

Miniati, F. 2015, ApJ, 800, 60

Miniati, F., \& Elyiv, A. 2013, ApJ, 770, 54

Miniati, F., Ryu, D., Kang, H., \& Jones, T. W. 2001, ApJ, 559, 59

Morlino, G., \& Caprioli, D. 2012, A\&A, 538, A81

Murase, K., Inoue, S., \& Nagataki, S. 2008, ApJ, 689, L105

Neronov, A., Semikoz, D., \& Vovk, I. 2010, A\&A, 519, L6

Neronov, A., \& Vovk, I. 2010, Science, 328, 73

Ohno, H., Takizawa, M., \& Shibata, S. 2002, ApJ, 577, 658

Palacio et al. 2015, ArXiv e-prints [arXiv: 1509.03974]

Pedlar, A., Ghataure, H. S., Davies, R. D., et al. 1990, MNRAS, 246, 477

Perkins, J. S. 2008, in AIP Conf. Ser., 1085, eds. F. A. Aharonian, W. Hofmann, \& F. Rieger, 569

Perkins, J. S., Badran, H. M., Blaylock, G., et al. 2006, ApJ, 644, 148

Petrosian, V. 2001, ApJ, 557, 560

Pfrommer, C. 2008, MNRAS, 385, 1242

Pfrommer, C., \& Enßlin, T. A. 2003, A\&A, 407, L73

Pfrommer, C., \& Enßlin, T. A. 2004a, A\&A, 413, 17

Pfrommer, C., \& Enßlin, T. A. 2004b, MNRAS, 352, 76

Pfrommer, C., Enßlin, T. A., \& Springel, V. 2008, MNRAS, 385, 1211

Pfrommer, C., Chang, P., \& Broderick, A. E. 2012, ApJ, 752, 24

Pinzke, A., \& Pfrommer, C. 2010, MNRAS, 409, 449

Pinzke, A., Oh, S. P., \& Pfrommer, C. 2015, ArXiv e-prints [arXiv: 1503.07870]

Pinzke, A., Pfrommer, C., \& Bergström, L. 2011, Phys. Rev. D, 84, 123509

Prokhorov, D. A., \& Churazov, E. M. 2014, A\&A, 567, A93

Reimer, O., Pohl, M., Sreekumar, P., \& Mattox, J. R. 2003, ApJ, 588, 155

Reiprich, T. H., \& Böhringer, H. 2002, ApJ, 567, 716

Rephaeli, Y., Nevalainen, J., Ohashi, T., \& Bykov, A. M. 2008, Space Sci. Rev., 134,71

Rolke, W. A., López, A. M., \& Conrad, J. 2005, Nucl. Instr. Methods Phys. Res. A, 551, 493

Ryle, M., \& Windram, M. D. 1968, MNRAS

Schlickeiser, R., Sievers, A., \& Thiemann, H. 1987, A\&A, 182, 21

Schlickeiser, R., Elyiv, A., Ibscher, D., \& Miniati, F. 2012a, ApJ, 758, 101

Schlickeiser, R., Ibscher, D., \& Supsar, M. 2012b, ApJ, 758, 102

Schlickeiser, R., Krakau, S., \& Supsar, M. 2013, ApJ, 777, 49

Selig, M., Vacca, V., Oppermann, N., \& Enßlin, T. A. 2015, A\&A, 581, A126

Sijbring, D., \& de Bruyn, A. G. 1998, A\&A, 331, 901

Sijbring, L. G. 1993, Ph.D. Thesis, Groningen University

Sironi, L., \& Giannios, D. 2014, ApJ, 787, 49

Supsar, M., \& Schlickeiser, R. 2014, ApJ, 783, 96

Takahashi, K., Mori, M., Ichiki, K., \& Inoue, S. 2012, ApJ, 744, L7

Tavecchio, F., Ghisellini, G., Foschini, L., et al. 2010, MNRAS, 406, L70

Tavecchio, F., Ghisellini, G., Bonnoli, G., \& Foschini, L. 2011, MNRAS, 414, 3566

Taylor, G. B., Gugliucci, N. E., Fabian, A. C., et al. 2006, MNRAS, 368, 1500

Taylor, A. M., Vovk, I., \& Neronov, A. 2011, A\&A, 529, A144

Vazza, F., \& Brüggen, M. 2014, MNRAS, 437, 2291

Vazza, F., Eckert, D., Brüggen, M., \& Huber, B. 2015, MNRAS, 451, 2198

VERITAS Collaboration 2009, ApJ, 706, L275

VERITAS Collaboration 2012, ApJ, 757, 123

Vestrand, W. T. 1982, AJ, 87, 1266

Vogt, C., \& Enßlin, T. A. 2005, A\&A, 434, 67

Voit, G. M. 2005, Rev. Mod. Phys., 77, 207

Völk, H. J., Aharonian, F. A., \& Breitschwerdt, D. 1996, Space Sci. Rev., 75, 279

Wiener, J., Oh, S. P., \& Guo, F. 2013, MNRAS, 434, 2209

Wik, D. R., Sarazin, C. L., Finoguenov, A., et al. 2011, ApJ, 727, 119

Wik, D. R., Sarazin, C. L., Zhang, Y.-Y., et al. 2012, ApJ, 748, 67 
Wik, D. R, Hornstrup, A. Molendi, S, et al. 2014, ApJ, 792, 48

Zandanel, F., \& Ando, S. 2014, MNRAS, 440, 663

Zandanel, F., Pfrommer, C., \& Prada, F. 2014, MNRAS, 438, 124

Zandanel, F., Tamborra, I., Gabici, S., \& Ando, S. 2015, A\&A, 578, A32

Zanin, M., Medina Alcazar, J., Carbajosa, J. V., et al. 2013, in Int. Cosmic Ray Conf., 0773

ZuHone, J. A., Markevitch, M., \& Johnson, R. E. 2010, ApJ, 717, 908

ZuHone, J. A., Markevitch, M., \& Lee, D. 2011, ApJ, 743, 16

ZuHone, J. A., Markevitch, M., Brunetti, G., \& Giacintucci, S. 2013, ApJ, 762, 78

ZuHone, J. A., Brunetti, G., Giacintucci, S., \& Markevitch, M. 2015, ApJ, 801, 146

1 ETH Zurich, 8093 Zurich, Switzerland

2 Università di Udine, and INFN Trieste, 33100 Udine, Italy

3 INAF National Institute for Astrophysics, 00136 Rome, Italy

4 Università di Siena, and INFN Pisa, 53100 Siena, Italy

5 Croatian MAGIC Consortium, Rudjer Boskovic Institute, University of Rijeka, University of Split and University of Zagreb, 51000 Rijeka, Croatia

6 Saha Institute of Nuclear Physics, 1/AF Bidhannagar, Salt Lake, Sector-1, 700064 Kolkata, India

7 Max-Planck-Institut für Physik, 80805 München, Germany

8 Universidad Complutense, 28040 Madrid, Spain

9 Inst. de Astrofísica de Canarias; Universidad de La Laguna, Dpto. Astrofísica, 38206 La Laguna, Tenerife, Spain

10 University of Łódź, 90236 Lodz, Poland

11 Deutsches Elektronen-Synchrotron (DESY), 15738 Zeuthen, Germany

12 Institut de Fisica d'Altes Energies (IFAE), The Barcelona Institute of Science and Technology, Campus UAB, 08193 Bellaterra (Barcelona), Spain

13 Universität Würzburg, 97074 Würzburg, Germany

14 Università di Padova and INFN, 35131 Padova, Italy

15 Centro de Investigaciones Energéticas, Medioambientales y Tecnológicas, 28040 Madrid, Spain

16 Institute for Space Sciences (CSIC/IEEC), 08193 Barcelona, Spain

17 Technische Universität Dortmund, 44221 Dortmund, Germany
18 Unitat de Física de les Radiacions, Departament de Física, and CERES-IEEC, Universitat Autònoma de Barcelona, 08193 Bellaterra, Spain

19 Universitat de Barcelona, ICC, IEEC-UB, 08028 Barcelona, Spain

20 Japanese MAGIC Consortium, ICRR, The University of Tokyo, Department of Physics and Hakubi Center, Kyoto University, Tokai University, The University of Tokushima, KEK, Japan

21 Finnish MAGIC Consortium, Tuorla Observatory, University of Turku and Department of Physics, University of Oulu, Finland

22 Inst. for Nucl. Research and Nucl. Energy, 1784 Sofia, Bulgaria

23 Università di Pisa, and INFN Pisa, 56126 Pisa, Italy

24 ICREA and Institute for Space Sciences (CSIC/IEEC), 08193 Barcelona, Spain

25 now at Centro Brasileiro de Pesquisas Físicas (CBPF/MCTI), R. Dr. Xavier Sigaud, 150 - Urca, RJ, 22290-180 Rio de Janeiro, Brazil

26 now at NASA Goddard Space Flight Center, Greenbelt, MD 20771, USA and Department of Physics and Department of Astronomy, University of Maryland, College Park, MD 20742, USA

27 Humboldt University of Berlin, Institut für Physik Newtonstr. 15, 12489 Berlin, Germany

28 Now at Ecole polytechnique fédérale de Lausanne (EPFL), 1015 Lausanne, Switzerland

29 Now at Department of Physics \& Astronomy, UC Riverside, CA 92521, USA

30 Also at Japanese MAGIC Consortium

31 Now at Finnish Centre for Astronomy with ESO (FINCA), 20740 Turku, Finland

32 Also at INAF-Trieste

33 Also at ISDC - Science Data Center for Astrophysics, 1290 Versoix (Geneva), Switzerland

34 Heidelberg Institute for Theoretical Studies, SchlossWolfsbrunnenweg 35, 69118 Heidelberg, Germany

35 Dark Cosmology Center, University of Copenhagen, Juliane Maries Vej 30, 2100 Copenhagen, Denmark

36 GRAPPA Institute, University of Amsterdam, 1098 XH Amsterdam, The Netherlands 